\title{
An empirical model of the global distribution of plasmaspheric hiss based on Van Allen Probes EMFISIS measurements
}

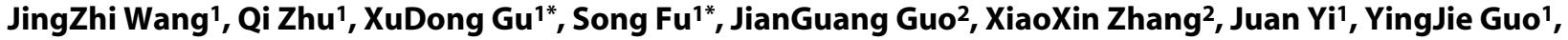 \\ BinBin $\mathrm{Ni}^{1}$, and Zheng Xiang ${ }^{1}$ \\ 'Department of Space Physics, School of Electronic Information, Wuhan University, Wuhan 430079, China; \\ ${ }^{2}$ Key Laboratory of Space Weather, National Center for Space Weather, China Meteorological Administration, Beijing 100081, China \\ Key Points: \\ - The empirical model is a third-order polynomial function of $L$-shell, magnetic local time (MLT), magnetic latitude (MLAT), and $A E^{*}$ \\ - The model can perform the hiss amplitude distribution by L/MLT and L/MLAT \\ - All data are based on Van Allen Probes EMFISIS measurements
}

Citation: Wang, J. Z., Zhu, Q., Gu, X. D., Fu, S., Guo, J. G., Zhang, X. X., Yi, J., Guo, Y. J., Ni, B. B., and Xiang, Z. (2020). An empirical model of the global distribution of plasmaspheric hiss based on Van Allen Probes EMFISIS measurements. Earth Planet. Phys., 4(3), $246-265$. http://doi.org/10.26464/epp2020034

\begin{abstract}
Using wave measurements from the EMFISIS instrument onboard Van Allen Probes, we investigate statistically the spatial distributions of the intensity of plasmaspheric hiss waves. To reproduce these empirical results, we establish a fitting model that is a thirdorder polynomial function of $L$-shell, magnetic local time (MLT), magnetic latitude (MLAT), and $A E^{*}$. Quantitative comparisons indicate that the model's fitting functions can reflect favorably the major empirical features of the global distribution of hiss wave intensity, including substorm dependence and the MLT asymmetry. Our results therefore provide a useful analytic model that can be readily employed in future simulations of global radiation belt electron dynamics under the impact of plasmaspheric hiss waves in geospace.
\end{abstract}

Keywords: hiss; Van Allen Probes; global model

\section{Introduction}

Plasmaspheric hiss is a broadband incoherent whistler mode emission with frequencies ranging from $\sim 20 \mathrm{~Hz}$ to $\sim 2 \mathrm{kHz}$ that is observed predominantly inside the plasmasphere or high density plumes (Thorne et al., 1973, 1979; Meredith et al., 2004; Li W et al., 2013, 2015a, b; Ni BB et al., 2014; Shi R et al., 2017). It can persist under quiet conditions; its fluctuations are positively correlated with the level of solar activity (Smith et al., 1974; Thorne et al., 1973 , 1974). Broadband amplitudes of plasmaspheric hiss range from a few pT to as high as 100 pT (Thorne et al., 1973; Smith et al., 1974; Meredith et al., 2004). Plasmaspheric hiss is mainly observed at a broad range of wave normal angle, e.g., its propagation near the geomagnetic equator is predominantly fieldaligned, but oblique at higher latitudes (Santolík et al., 2001; Bortnik et al., 2008).

It has been well recognized that electron scattering by plasmaspheric hiss acts as a dominant contributor to formation of the slot region, which separates the radiation belts into inner $(1.2<L<2)$ and outer $(3<L<7)$ parts (Lyons et al., 1972; Lyons

\section{Correspondence to: X. D. Gu, guxudong@whu.edu.cn}

$$
\text { S. Fu, fusion@whu.edu.cn }
$$

Received 20 JAN 2020; Accepted 02 MAR 2020.

Accepted article online 08 MAY 2020.

(C) 2020 by Earth and Planetary Physics. and Thorne, 1973; Albert, 1994; Abel and Thorne, 1998a, b; Meredith et al., 2004, 2006a, b). Resonant interactions with plasmaspheric hiss result in electron scattering losses with decay time scales varying from less than 1 hour to tens or hundreds of days. Decay times are closely dependent on electron energy, ambient magnetic field, and plasma density, as well as on wave amplitude, spectral intensity, and wave normal angle (WNA) distribution of the hiss waves as a function of spatial location and geomagnetic activity level (Meredith et al., 2007; Ni BB et al., 2014; Yu J et al., 2017). As reported in Summers et al. (2007a, b), the pitch angle diffusion coefficient of resonant electrons in quasi-linear formalisms is proportional to the wave amplitude. Thus, the development of a reliable global model of hiss wave amplitude is essential to facilitate quantification of hiss-induced electron scattering rates and resultant global variations of radiation belt electron distribution.

A number of empirical models of hiss wave global distribution have been constructed in previous studies (Meredith et al., 2004; Kim et al., 2015; Li et al., 2015b). Data from the Combined Release and Radiation Effect Satellite (CRRES) are utilized to investigate the features of plasma waves. Meredith et al. (2004) presented elaborate global maps of hiss wave intensity and described its distribution features. Orlova et al. (2014) produced empirical quadratic fittings of $B_{\mathrm{w}}^{2}$ as a function of $K p, L$, and geomagnetic latitude $(\lambda)$ for the daytime and nighttime sectors respectively. However, 
the CRRES wave instrument has limitations in space and frequency band, in particular it records only electric field data; accordingly, some important wave information such as magnetic field spectral intensity was estimated, based on theoretical assumptions. Characteristics of whistler mode waves have also been analyzed by Agapitov et al. (2013) with Cluster data, which separate chorus from hiss merely by $f_{\text {ce }}$ (the electron cyclotron frequency), i.e., chorus frequency above $0.1 f_{\text {ce }}$ and hiss below $0.1 f_{\text {ce, }}$ though their frequencies may actually overlap. Subsequently Agapitov et al. (2014) parameterized the hiss wave activity at $L<2$ based on Akebono spacecraft observations. In addition, combined observations of Cluster (Agapitov et al., 2013) and Polar (Tsurutani et al., 2015) showed that hiss can be widely distributed over the magnetic latitude, extending to $\lambda>45^{\circ}$.

Recently, using data from Van Allen Probes, Spasojevic et al. (2015) and $\mathrm{Yu} J$ et al. (2017) constructed one-dimensional fitting models of hiss wave amplitude as a function of $A E, L, M L T$, and $K p$, $L, M L T$, respectively. Highlighting the dependence of hiss amplitude on single variables, those studies focus on the fluctuations of the fitting curves associated with each variable in their hypothetical formulae and do not reproduce the observed latitudinal variations of hiss wave amplitude. Since the dependence on MLAT cannot be neglected in accurate predictions of hiss amplitude, as previous studies have done, the present investigation considers the combined effects of $L, M L T, M L A T$, and $A E^{*}$, and intends to establish a more comprehensive empirical model of plasmaspheric hiss amplitude as a function of these four input parameters.

\section{Data and Methodology}

Van Allen Probes were launched on September 8, 2012 with perigee of $\sim 1.1 R_{\mathrm{E}}$ (radius of the Earth), apogee of $\sim 5.8 R_{\mathrm{E}}$, inclination of $10^{\circ}$, and an orbital period of $\sim 9$ hours. Equipped with identical electromagnetic detectors, the EMFISIS instruments onboard Van Allen Probes can acquire high quality measurement of whistlermode waves in the inner magnetosphere (Kletzing et al., 2013). The waveform receiver (WFR) on EMFISIS provides wave power spectral densities ranging from $10 \mathrm{~Hz}-12 \mathrm{kHz}$ with a temporal resolution of $6 \mathrm{~s}$. The high frequency receiver (HFR) records electric spectral information between 10 and $400 \mathrm{kHz}$, in which the traces of upper hybrid resonance (UHR) frequency can be used to estimate the ambient plasma density (Mosier et al., 1973) and identify the location of the plasmapause that separates the regions outside and inside the plasmasphere (e.g., He F et al., 2011, 2013, 2016, 2017; Katus et al., 2015; Verbanac et al., 2015; Zhang XX et al., 2017a, b).

According to the wave characteristics of hiss emissions, in this study we identify a hiss event with the ellipticity $>0.7$ (i.e., righthand polarized) and the frequency band within $10-2000 \mathrm{~Hz}$ in the plasmasphere. By doing so, we distinguish the hiss waves from chorus waves outside the plasmasphere and from magnetosonic waves with nearly linear polarization. Subsequently, we integrate the wave spectral intensity in the determined frequency band to calculate the wave amplitude $\left(B_{\mathrm{w}}\right)$. In our following analysis we concentrate on the hiss emissions with $B_{\mathrm{w}} \geq 5 \mathrm{pT}$.

The adopted database consists of observations made during the period from September 8, 2012 to June 30, 2017: L-shell (L), magnetic local time (MLT), magnetic latitude (MLAT), $A E^{*}$ (averaged value of the $A E$ index in the previous hour), and hiss wave amplitude $\left(B_{\mathrm{w}}\right)$ with temporal resolution of $6 \mathrm{~s}$. We then implement two methods to construct our empirical global model of hiss wave amplitude. In each method, we divide the entire database data into two groups, i.e., the training group and the test group. The training group is used as the baseline to obtain the fitting coefficients in the regression, while the test group is regarded as a comparison to examine the model's performance and verify its reliability.

As tabulated in Table 1, for Method \#1, data in first three years are selected as the training group (Database 1) and the remaining datasets are reserved as the test group (Database 2). For Method $\# 2$, we divide them in another way: data collected during oddnumber days are set as the training group (Database 3 ), and the others are the test group (Database 4). Furthermore, we divide each Database (1-4) into 12 sub-groups in terms of $A E^{*}$ and MLT, to take into account the geomagnetic effect and MLT variation. Hence, we rank the $A E^{*}$ values into three geomagnetic levels (quiet, $A E^{*}<100 \mathrm{nT}$; moderate, $100 \mathrm{nT} \leq A E^{*} \leq 300 \mathrm{nT}$; active, $A E^{*}$ $>300 \mathrm{nT}$ ) and classify all the MLTs into four sectors (dayside, 09-15; duskside, 15-21; nightside, 21-03; dawnside, 03-09). Correspondingly, twelve groups of fitted parameters need to be derived from regression of these training groups, and we then examine the feasibility of the resulting fitting functions through selfconsistency and verification with the test groups. To check differences between the model and observations, we define the variable, $R_{d}$, given as $\frac{\left|w_{\text {obs }}-w_{\text {mod }}\right|}{w_{\text {obs }}}$, where $w_{\text {obs }}$ is the observed hiss wave amplitude and $w_{\text {mod }}$ is the model result, as a criterion to evaluate our empirical model. Clearly, lower values of $R_{d}$ represent higher similarity between observations and the model.

Taking $L, M L T, M L A T, A E^{*}$, and $B_{\mathrm{w}}$ as known quantities and $F(i, j, k$, I) as unknown, we implement the fits of a third-order polynomial function as follows:

$$
\log _{10}\left(B_{w}\right)=\sum_{i=0}^{3} \sum_{j=0}^{2} \sum_{k=0}^{2} \sum_{l=0}^{2} F(i, j, k, l) A^{i} B^{j} C^{k} D^{\prime},
$$

where the parameters $A, B, C$, and $D$ are quantified as $L / 10$, $\mathrm{MLT} / 24, \mathrm{MLAT} / 20$, and $A E^{*} / 500$, respectively. Following previous studies of Spasojevic et al. (2015) and Yu et al., (2017), we set the

Table 1. Classification of each adopted database.

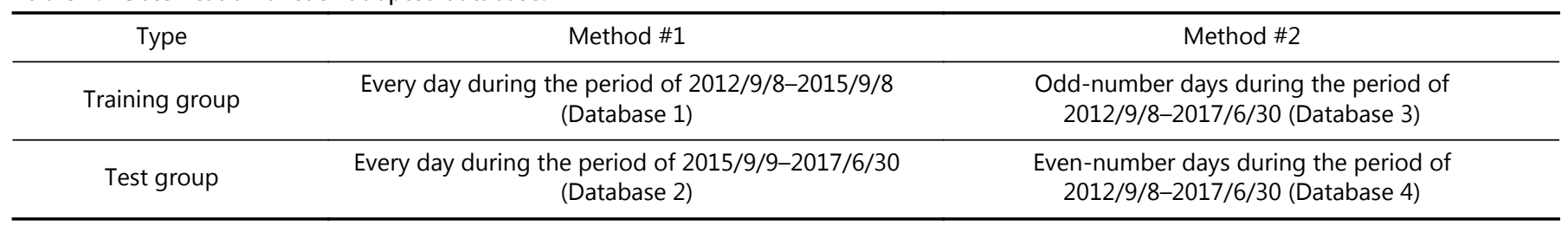


maximum values of $i, j, k$, and $/$ as $3,2,2$, and 2, respectively; consequently we have 108 coefficients for the fit to each training group.

\section{Analysis Results}

Based on the two methods described above, we do the fits for the training groups, compute the model results with the obtained polynomial fitting functions, and perform quantitative comparisons with the statistical observations of the test groups.

First, we follow Method \#1 to establish the empirically analytic model of plasmaspheric hiss intensity, the results of which are shown in Figures 1-4. Figures 1 and 2 show the global distributions of time-averaged root-mean-square (RMS) hiss wave amp- litudes in the equatorial plane and the meridian plane, at resolution of $0.5 \mathrm{~L} \times 1 \mathrm{MLT}$ and $1^{\circ} \times 0.1 \mathrm{~L}$, respectively, obtained using Database 1 (the period from 2012/9/8-2015/9/8), and comparisons between these statistical observations and results of the empirical model.

The first-row subplots in Figure 1 reveal that the hiss wave amplitude strengthens significantly when $A E^{*}$ increases and that the waves on the dayside are much stronger than those at the nightside. Overall, hiss wave activity shows a strong dependence on $L$ shell, MLT, and geomagnetic level. Correspondingly, the secondrow panels illustrate model results of hiss wave amplitude for the same time period as in Figures $1 \mathrm{a}-1 \mathrm{c}$, obtained using the fitted three-order polynomial function (i.e., Equation (1)). It is evident

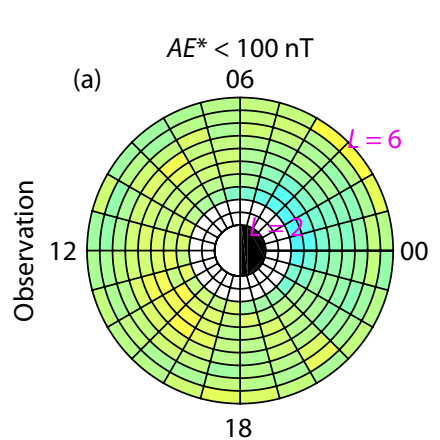

Database 1: 2012/9/8-2015/9/8

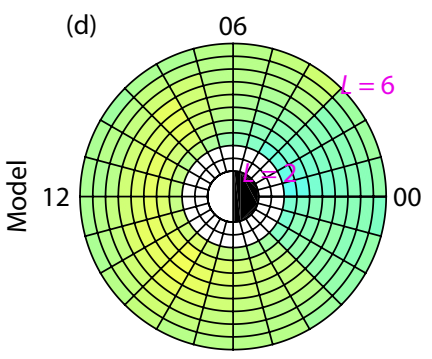

$100 \mathrm{nT} \leq A E^{*} \leq 300 \mathrm{nT}$
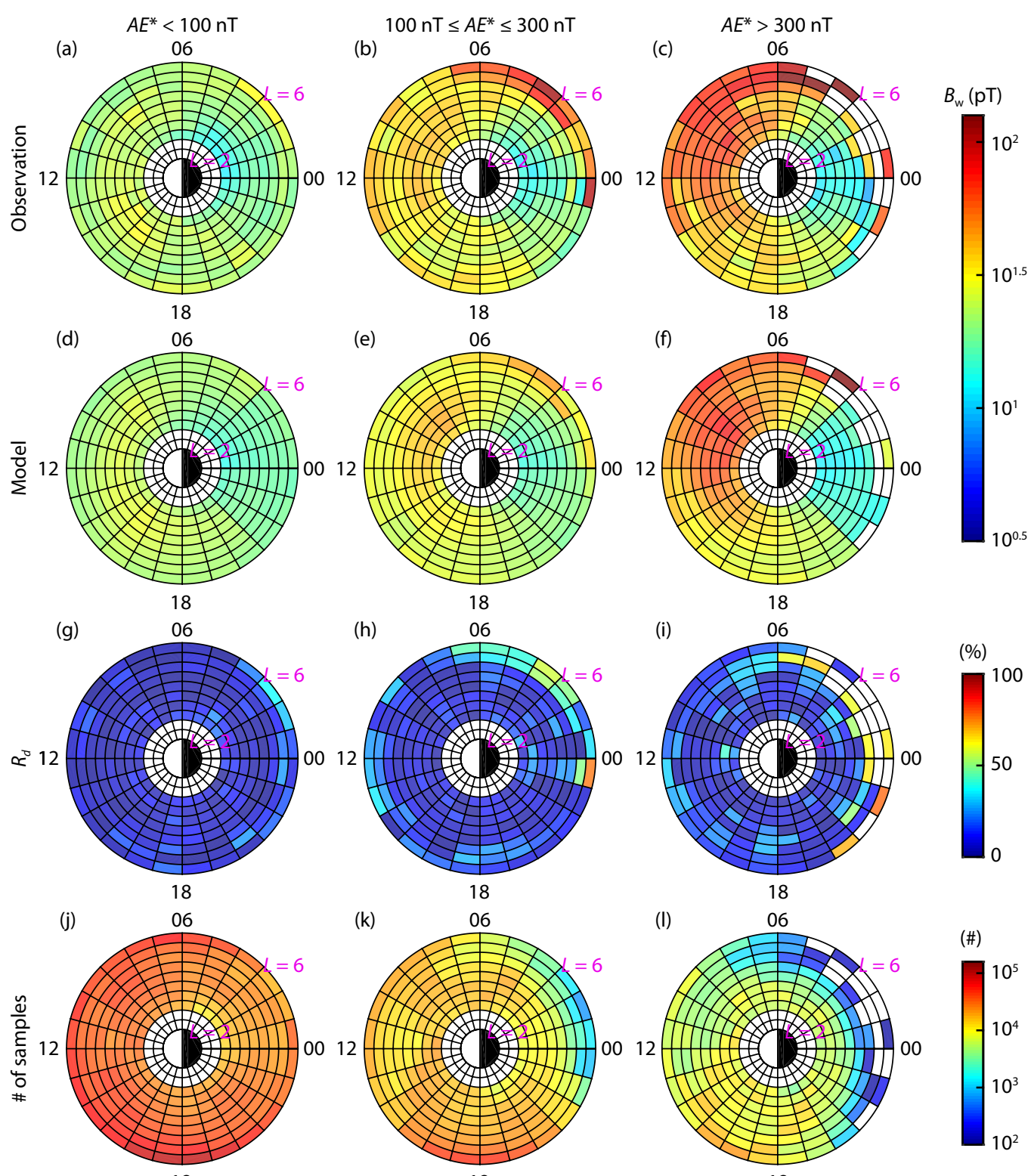

(\#)

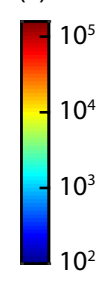

18

Figure 1. (a-c) Global L-MLT distribution of hiss wave amplitude from Van Allen Probe observations of Database 1; ( $d-f)$ self-consistent model results of Database $1 ;(\mathrm{g}-\mathrm{i})$ relative differences $\left(R_{d}\right)$ between them; $(\mathrm{j}-\mathrm{l})$ corresponding numbers of samples collected during quiet, moderate, and active times (left to right). 

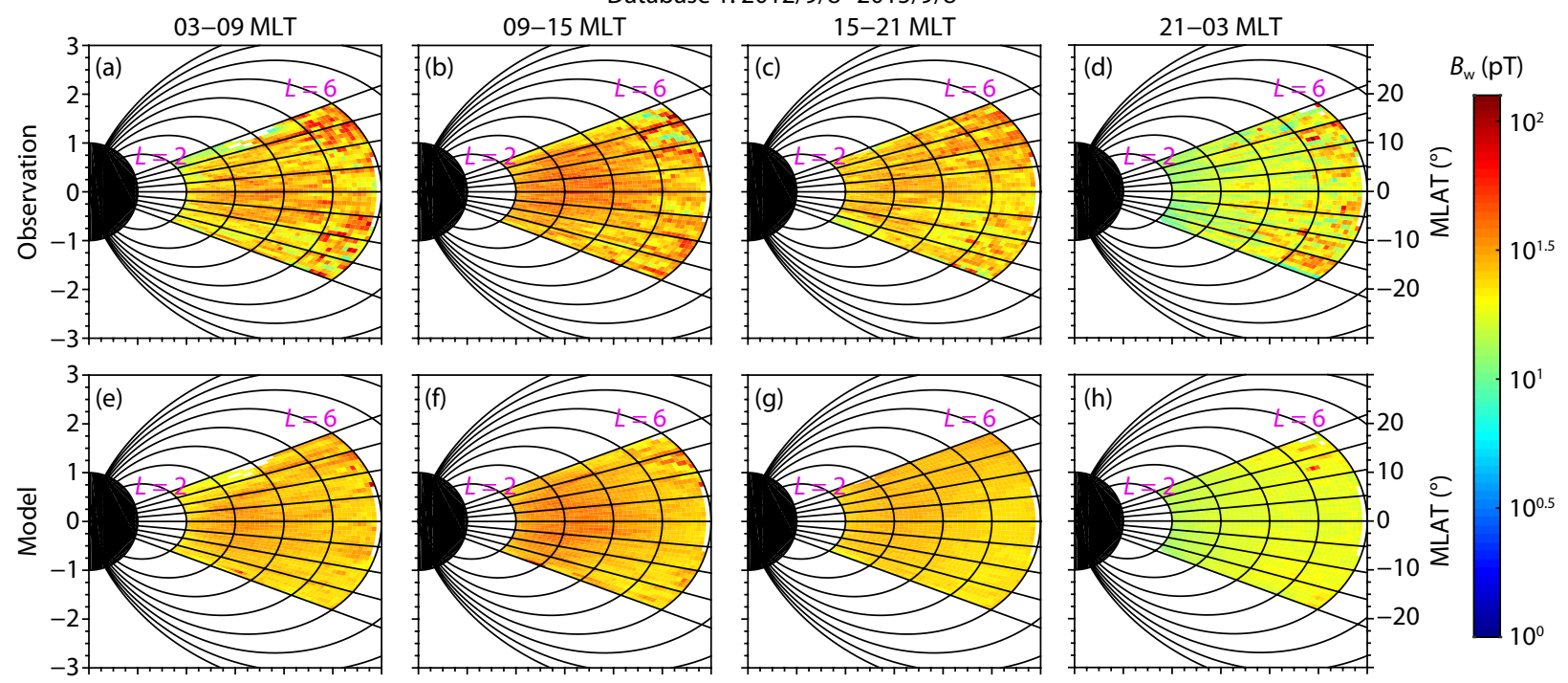

$-10^{1}$
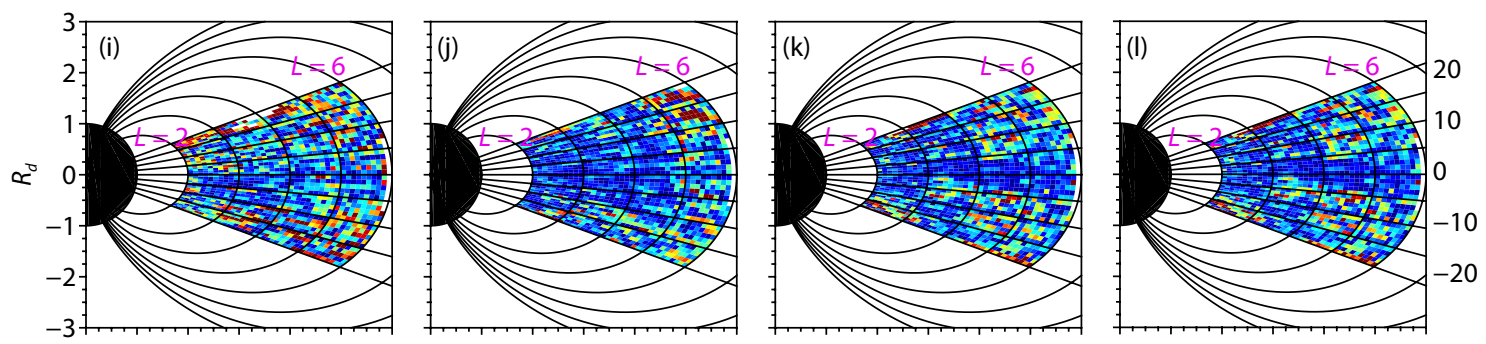

(\%)
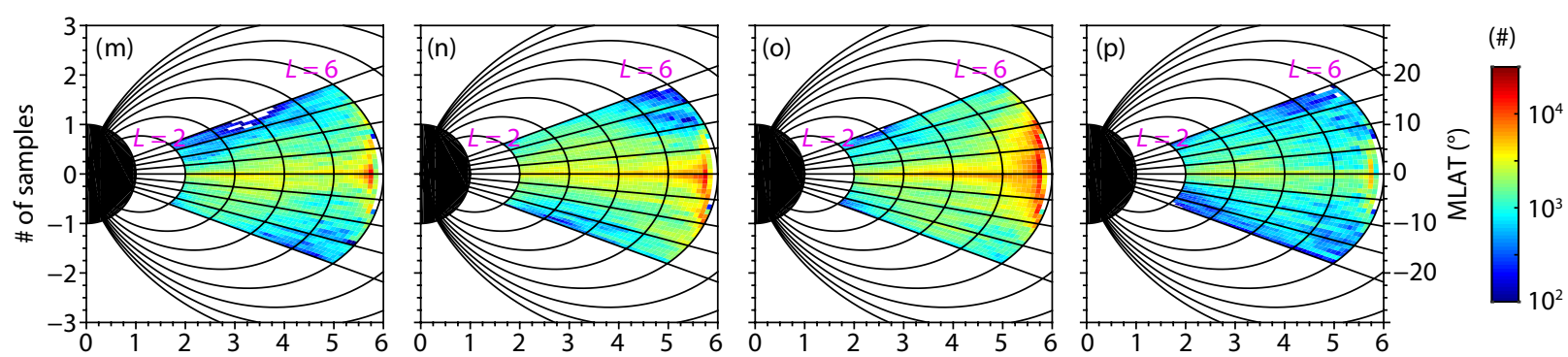

Figure 2. (a-d) Latitudinal L-MLAT distribution of hiss wave amplitude from observations in Database 1 ; (e-h) self-consistent model results of Database $1 ;(\mathrm{i}-\mathrm{l})$ relative differences $\left(R_{d}\right)$ between them; $(\mathrm{m}-\mathrm{p})$ corresponding numbers of samples during different MLT sectors (left to right).

that the fitted function reflects the major features of the observed global dynamics of plasmaspheric hiss. The relative differences $\left(R_{d}\right)$ between the observations and model results are shown in Figures $1 \mathrm{~g}-1 \mathrm{i}$. It is clearly seen that during most bins the relative differences are less than $15 \%$, though the differences are larger in some limited regions of $L=4.5-6$ and MLT 23-06.

Figure 2 results are similar to those in Figure 1 but as a function of $L$ and MLAT in the indicated four MLT sectors. It can be seen clearly that, besides its $L$-shell and MLT dependence, the hiss wave amplitude also depends on MLAT but in a less significant manner. Figures $2 \mathrm{i}-2 \mathrm{l}$ confirm that during most bins the relative differences between the observations and model results are less than $20 \%$, showing good agreement between model and empirical data.

Figures 3-4 present Database 2 (the test data) results in the same format as Figures $1-2$, to verify consistent model performance during periods exclusive to the training group.

While the wave observations are slightly different from those in
Database 1, the dominant trends are favorably similar. In order to validate the feasibility of the fitting function, we compare the observations from Database 2 (Figures 3a-3c) with the model results for the same period that are obtained using the empirically analytic function model as a function of $L, M L T, M L A T$, and $A E^{*}$ derived using Database 1 (Figures 3d-3f). Apparently, large differences between the observational and model results occur especially during geomagnetically moderate and active times, showing that the value of $R_{d}$ can be well above 1 in association with large deviation of the model results from the observations. In addition, it is distinct in Figure 4 that the model results become much less reliable compared to the observations, especially for the interval of MLT = 03-09. These discrepancies between model results and observations during the time period exclusive to the test group tell that the empirical model obtained using Method $\# 1$ has limitations that make it unsuited to the task of obtaining a global hiss wave distribution.

Accordingly, we adopt Method \#2 to establish an empirical model of the global distributions of hiss intensity; that is, the data during 


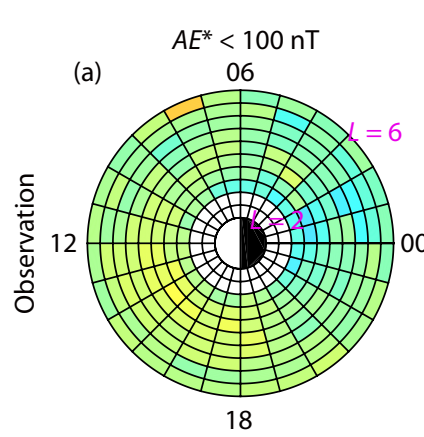

Database 2: 2015/9/9-2017/6/30
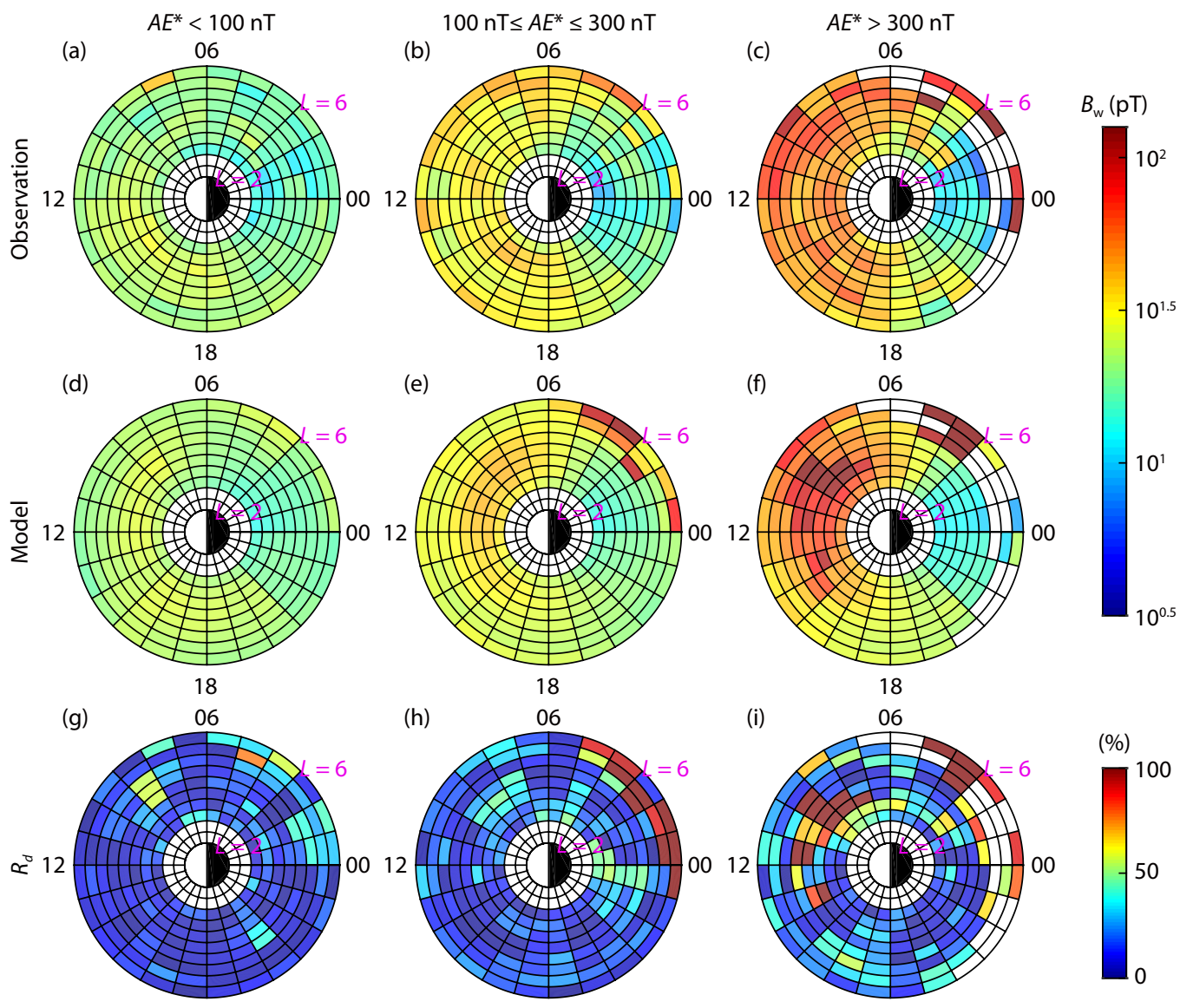

18
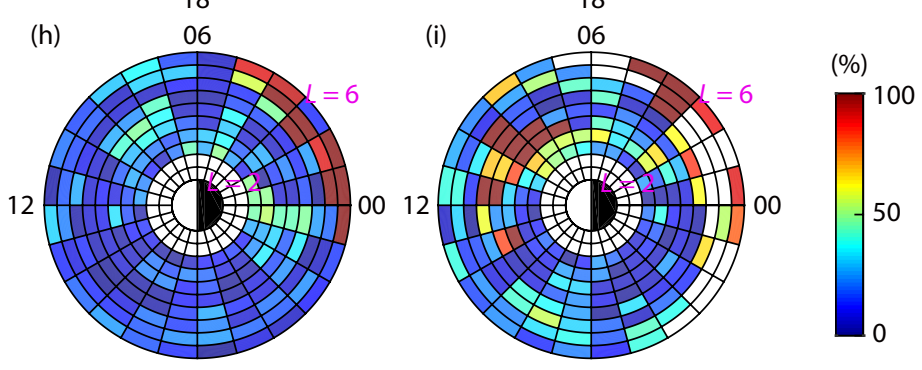

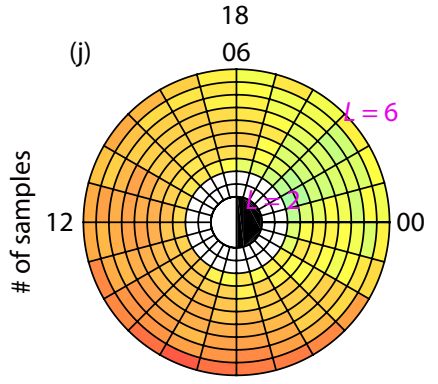

18

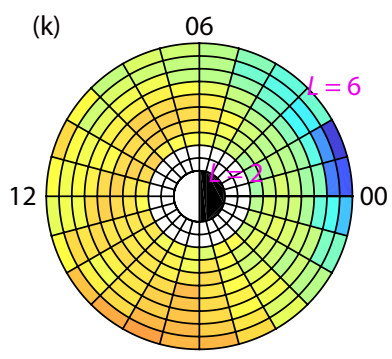

18

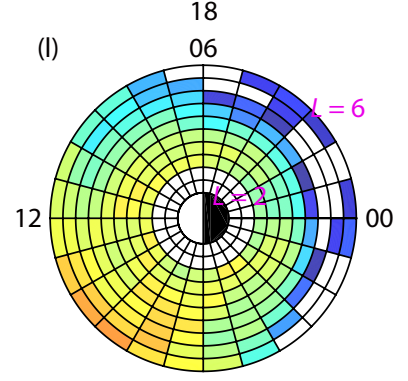

18

Figure 3. $(a-c)$ Global L-MLT distribution of hiss wave amplitude from observations of Database 2; (d-f) model results calculated by Method \#1 of Database $2 ;(\mathrm{g}-\mathrm{i})$ relative differences $\left(R_{d}\right)$ between them; $(\mathrm{j}-\mathrm{l})$ corresponding numbers of samples during quiet, moderate, and active times (left to right).

the odd-number days are set as the training group (Database 3) and used to acquire the fitting function $F(i, j, k, l)$, and the data during the even-number days are set as the test group to verify the model's performance.

First, we use Database 3 for the fits and self-consistency comparisons, the results of which are shown in Figures 5 and 6. Evidently, the fitting model results and the observations exhibit favorable agreement in most L-MLT bins (Figure 5), justifying the feasibility of the empirical model. While the differences between the observations and the model become relatively larger in the $L$-MLAT bins at high $L$ shell, the majority of $R_{d}$-values are below $\sim 20 \%$ (Figure 6), thereby confirming good performance of the model.
Again, to validate the performance of the fitting model obtained using Database 3 as the training group, we use Database 4 to apply the model and compare the model results with the observations, the results of which are shown in Figures 7 and 8.

Compared with the statistical observations (Figures 7a-7c), the model can reproduce well the global distribution of hiss wave amplitude including the MLT asymmetry and geomagnetic activity dependence (Figures 7d-7f). As shown in Figures 7g-7i, the relative differences $\left(R_{d}\right)$ are $<15 \%$ for over $80 \%$ of $L$-MLT bins, justifying good performance of the fitting model, while the model becomes less feasible at high $L$-shells on the nightside, especially for active conditions with $A E^{*}>300 \mathrm{nT}$. In addition, the empirical model can reproduce well the major features of hiss wave amp- 

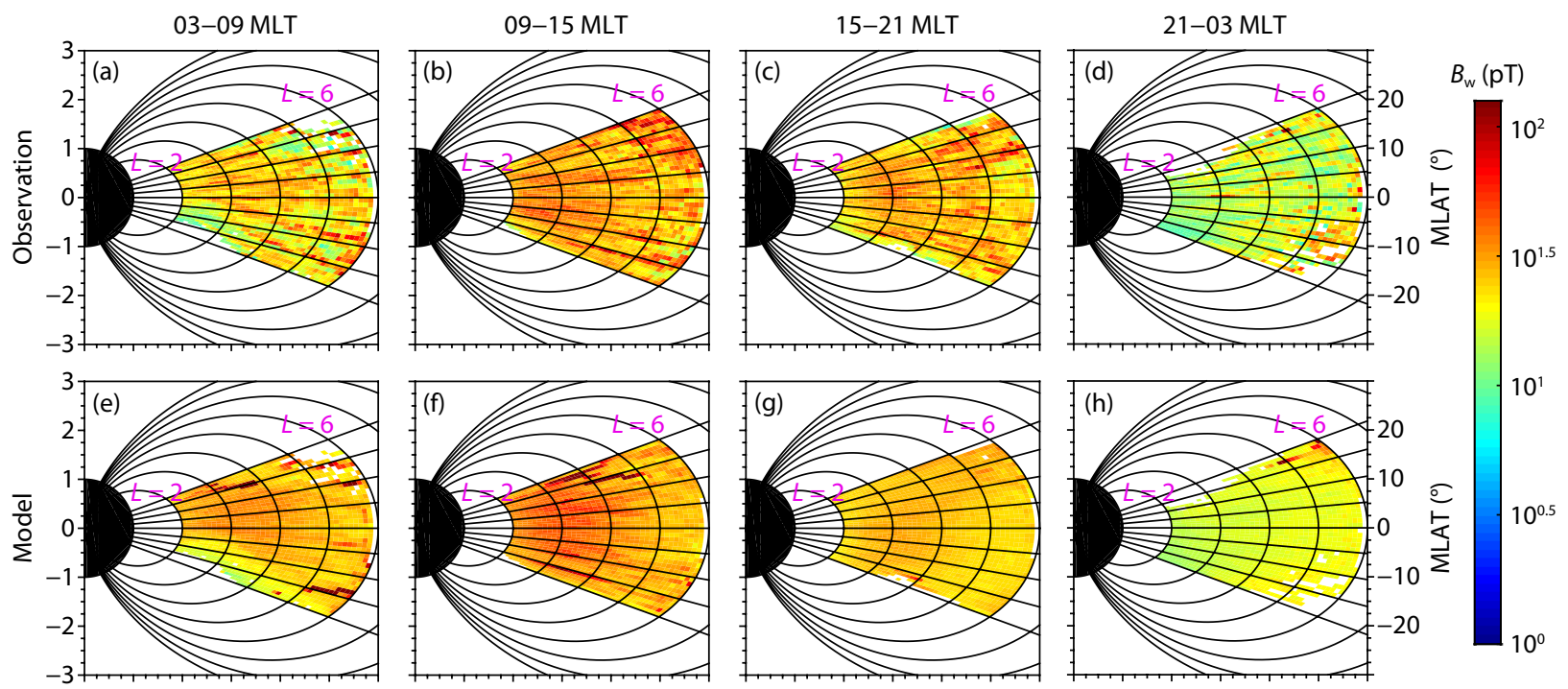

10
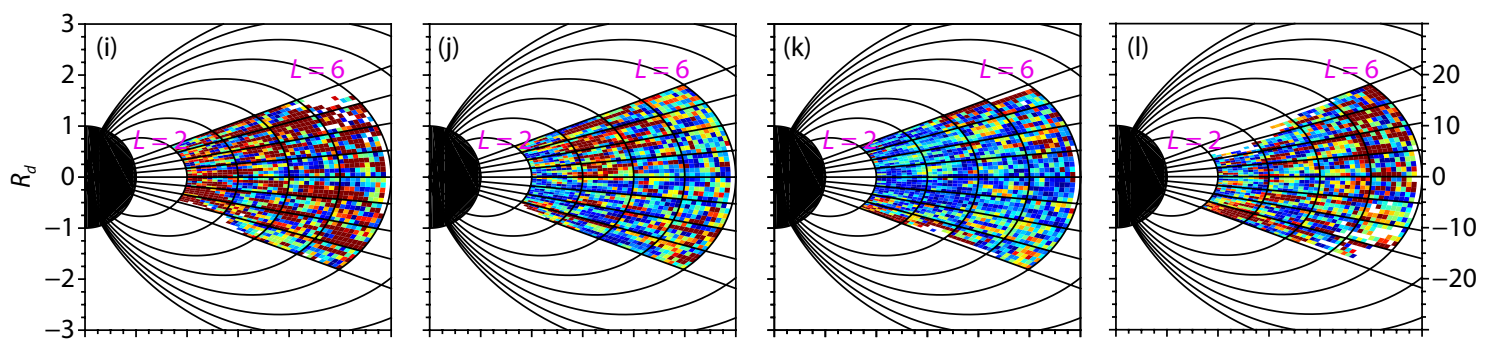

(\%)
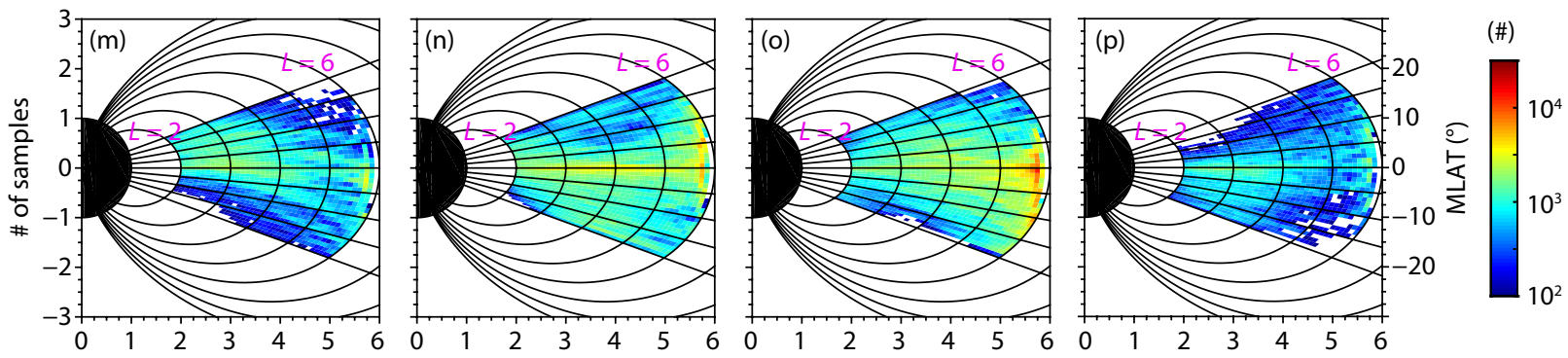

Figure 4. (a-d) Latitudinal L-MLAT distribution of hiss wave amplitude from observations in Database 2; (e-h) model results calculated by Method \#1 of Database $2 ;(\mathrm{i}-\mathrm{l})$ relative differences $\left(R_{d}\right)$ between them; $(\mathrm{m}-\mathrm{p})$ corresponding numbers of samples during different MLT sectors (left to right).

litude distribution in the L-MLAT bins for different MLT intervals, as shown by the $R_{d}$-values $<20 \%$ in general (Figures $8 \mathrm{i}-8 \mathrm{I}$ ).

Some discrepancies at higher latitudes may be attributed to hiss wave amplitude enhancements during geomagnetically disturbed periods, which, however, are difficult to be captured by the fits possible with third-order polynomial functions.

Overall, this study establishes an empirical model of the global distribution of hiss wave intensity by implementing third-order polynomial function fits to the long-term Van Allen Probes EMFISIS wave data, which is determined by Equation (1) and the polynomial coefficients tabulated in Tables S1-S12 in the Appendix for the four considered MLT sectors (i.e., 03-09, 09-15, 15-21, and 21-03) under three geomagnetic conditions (i.e., quiet-time: $A E^{*}<100 \mathrm{nT}$; moderate-time: $100 \mathrm{nT} \leq A E^{*} \leq 300 \mathrm{nT}$; active-time: $A E^{*}>300 \mathrm{nT}$ ).

\section{Concluding Remarks}

In the present study, we have used approximately five years of Van Allen Probes EMFISIS wave data to analyze statistically the global distribution of plasmaspheric hiss intensity at $L=2-6$ under various conditions of geomagnetic activity. During quiet times or times of weak substorm activity $\left(A E^{*}<100 \mathrm{nT}\right)$, the hiss wave amplitude distribution is not dependent on MLT. However, when substorm activity intensifies, the hiss wave amplitude distribution is more dependent on MLT and the intense hiss events are concentrated at high $L(L>5)$ on the morning side and the nightside (MLT 00:00-06:00). Furthermore, the observation results (the first row of our Figures 2, 4, 6, and 8) suggest that the hiss amplitude distribution is slightly dependent on MLAT in the data on which the present study is based, which means the distribution is mainly controlled by $L$ and MLT. The hiss wave amplitude is very intense inside the dayside plasmasphere (the first row of Figures 


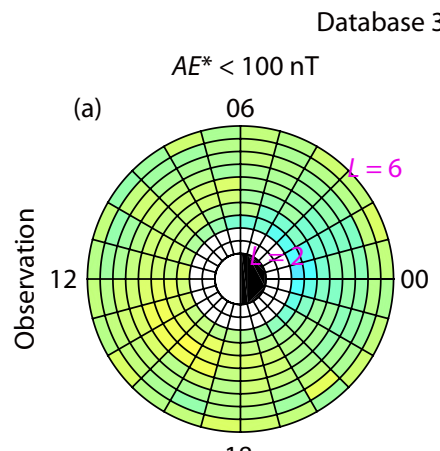

18

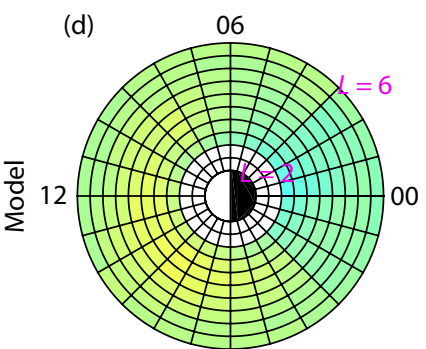

18

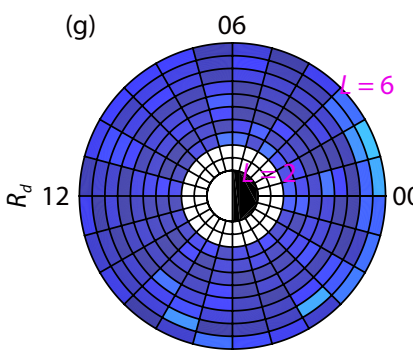

18

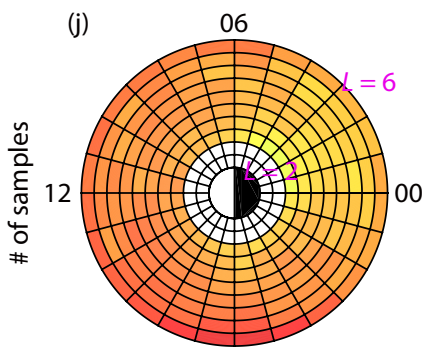

18

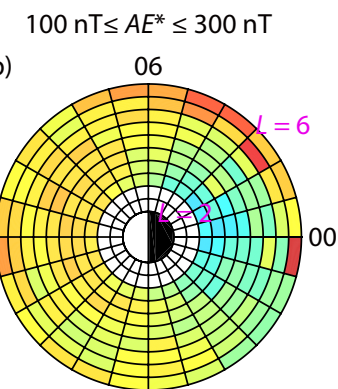

18

(e)

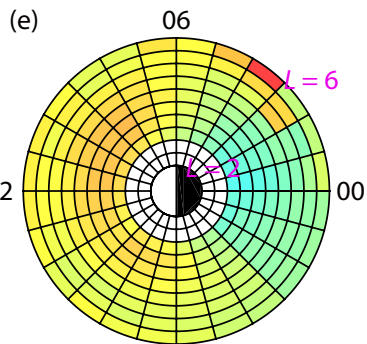

18

(h)

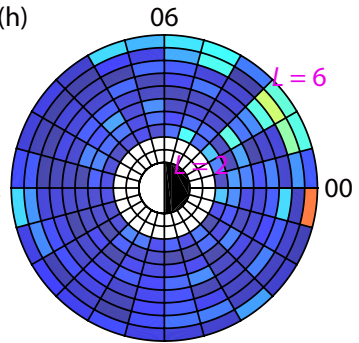

18

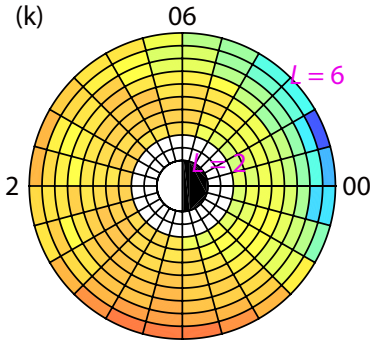

18

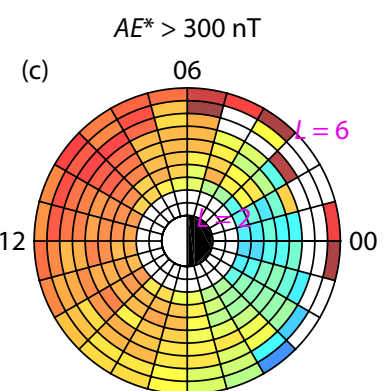

18

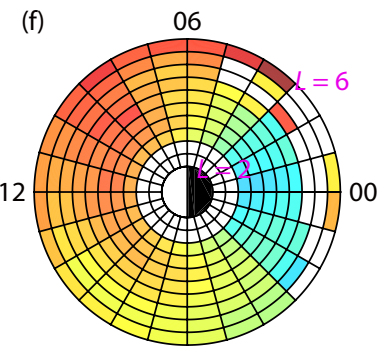

18

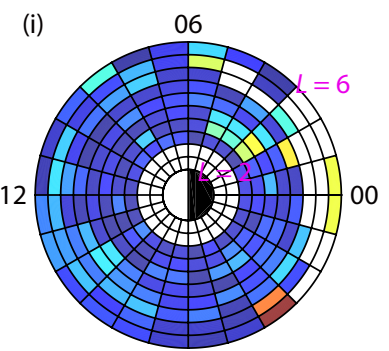

18

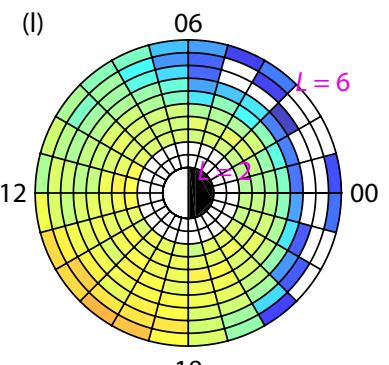

18

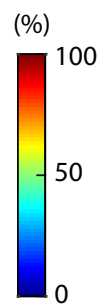

(\#)

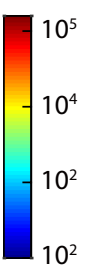

Figure 5. $(a-c)$ Global L-MLT distribution of hiss wave amplitude from observations of Database 3; ( $d-f$ ) self-consistent model results of Database $3 ;(\mathrm{g}-\mathrm{i})$ relative differences $\left(R_{d}\right)$ between them; $(j-\mathrm{l})$ corresponding numbers of samples collected during quiet, moderate, and active times (left to right).

$1,3,5$, and 7). Inspired by observations that hiss wave amplitudes vary sensitively with spatial location and $A E^{*}$, we have then performed numerical fits to the statistical observations in terms of a third-order polynomial as a function of $L, M L T, M L A T$, and $A E^{*}$. By doing so, we have constructed an empirically analytic model of the global distribution of plasmaspheric hiss amplitude. The significance mainly lies in the treatment of fluctuation. The hiss amplitude can be obtained through the model simply by using $A E^{*}$ and position information. The results of previous studies (Spasojevic et al., 2015, Yu J et al., 2017) show good agreement with hiss amplitude observations when for single variables ( $L$ or MLT) are considered. Since the 2D model including L/MLT or L/MLAT is of greater importance, in this study we present the fitting model to investigate the relationship between $B_{\mathrm{w}}$ and $L / \mathrm{MLT}$, and also $B_{\mathrm{w}}$ and L/MLAT. Our 2D model can perform the hiss amplitude distribution by L/MLT and L/MLAT simultaneously. Beyond this, our model can be simplified for 1D usage; the results show good consistency with Spasojevic et al. (2015) and Yu J et al. (2017).

Two different methods have been implemented to separate the training group and the test group, as shown in Table 1. Our analysis indicates that Method \#2, which uses the odd-number days as the training group and the even-number days as the test group, is superior in deriving well-performing fitting functions for the global hiss wave model. One possible reason can be that the training and test groups in Method \#2 may reflect the phases of a solar cycle more reasonably. Several other factors that may affect the modeling results should also be considered: first, the latitudin- 


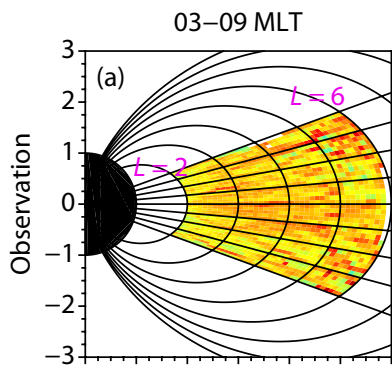

Database 3: 2012/9/8-2017/6/30 (odd number of days)
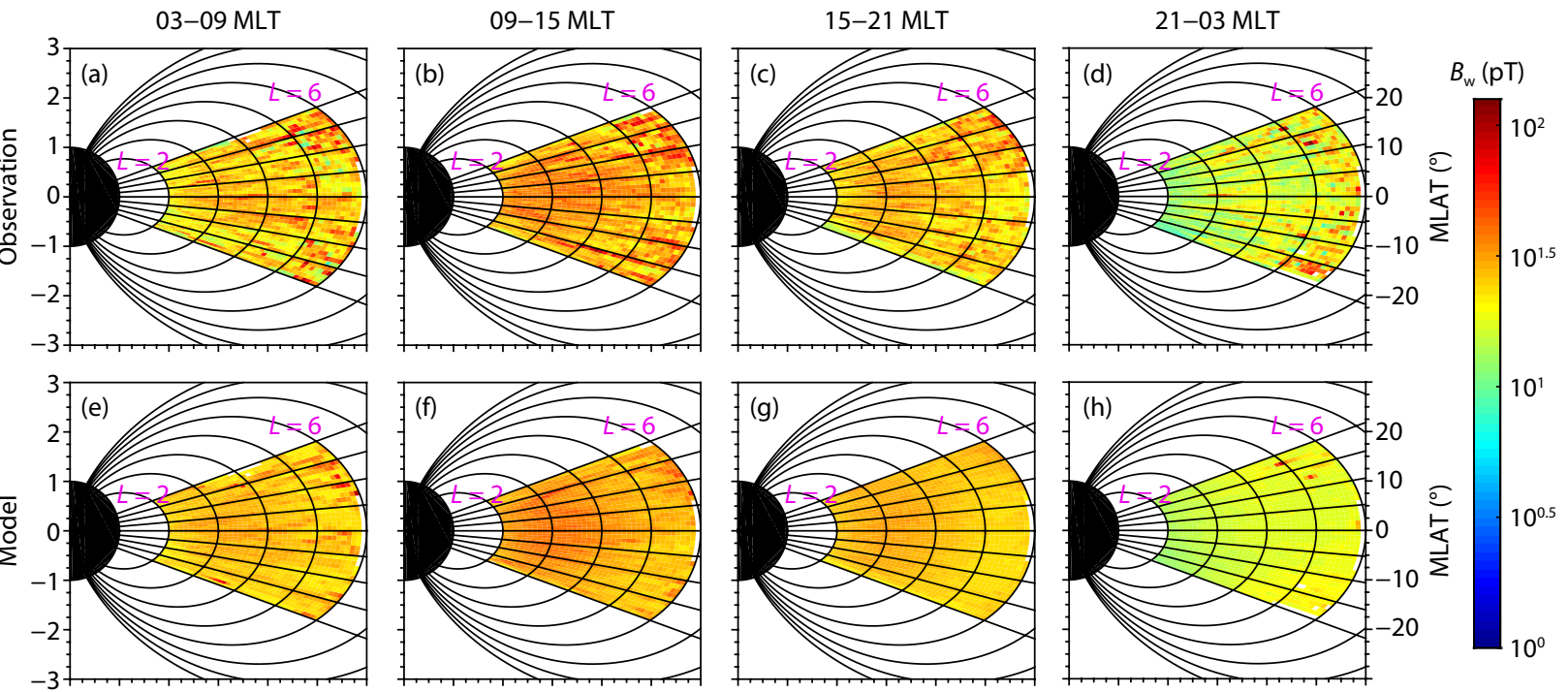

$-10^{1}$
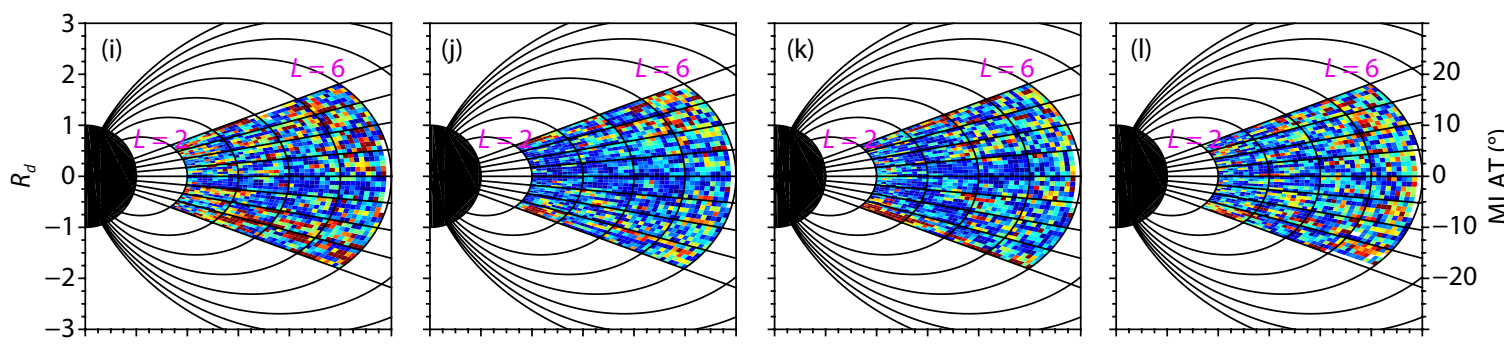

(\%)
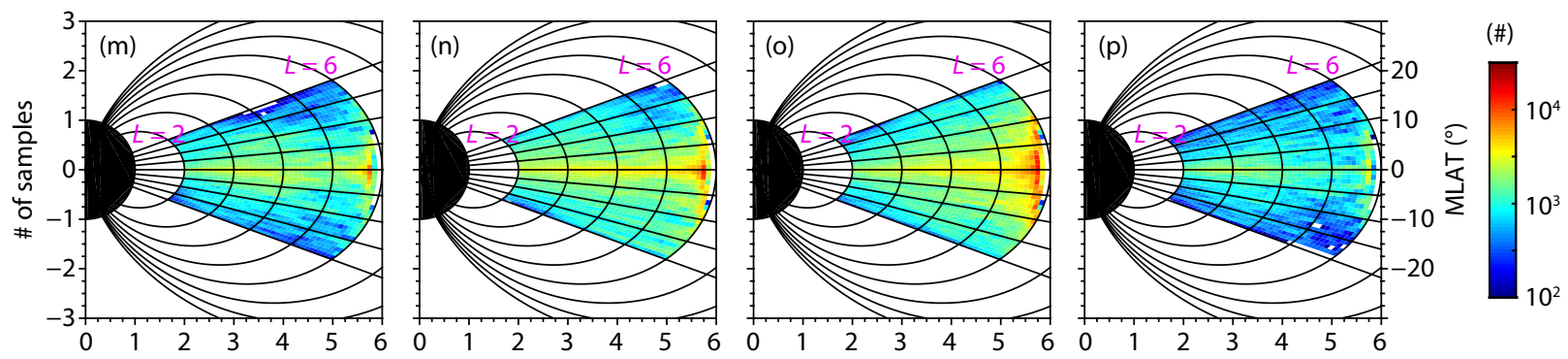

Figure 6. (a-d) Latitudinal L-MLAT distribution of hiss wave amplitude from observations in Database 3; (e-h) self-consistent model results of Database 3 ; $(\mathrm{i}-\mathrm{l})$ relative differences $\left(R_{d}\right)$ between them; $(\mathrm{m}-\mathrm{p})$ corresponding numbers of samples during different MLT sectors (left to right).

al distribution of the number of samples is dependent on the MLAT, which could be another reason why Method \#1 is worse than Method \#2; second, Databases 1 and 2 have more differences in the MLAT distribution, which may also be the cause of differences between the observations and model results. These unsolved questions require further investigation in future studies.

In summary, it is well demonstrated, by quantitative comparisons between the statistical observations and model results, that the empirical model in terms of fitted functions can reflect favorably the major features of the global distribution of hiss wave intensity, including the MLT asymmetry, substorm dependence, and latitudinal variations (Xiang $Z$ et al., 2017). Because both the energy spectra and pitch angle distributions of radiation belt electrons are critically affected by hiss wave scattering (e.g., Ni BB et al., 2013, 2014, 2017, 2019; Zhao H et al., 2019; Li LY et al., 2008; Hua $M$ et al., 2019), and because the scattering rates increase proportionally to the square of hiss amplitude for the near-resonance cases, our results therefore provide an empirically useful analytic model to be readily used for numerical quantification of hiss-driven electron diffusion coefficients and global simulations of resultant modulation of radiation belt electron dynamics in response to varying conditions of solar wind and geomagnetic activities.

\section{Acknowledgments}

This work was supported by the B-type Strategic Priority Program of the Chinese Academy of Sciences (Grant No. XDB41000000), the NSFC grants 41674163, 41974186, 41704162, 41904144, and 41904143, and the pre-research projects on Civil Aerospace Technologies No. D020308, D020104 and D020303 funded by China National Space Administration. We thank the Van Allen Probes EMFISIS Science Team for providing the data. The EMFISIS data are obtained from EMFISIS website (https://emfisis.physics. uiowa.edu/data/index); the geomagnetic activity indices are obtained from the NASA OMNIWeb (http://cdaweb.gsfc.nasa.gov). 
Database 4: 2012/9/8-2017/6/30 (even number of days)
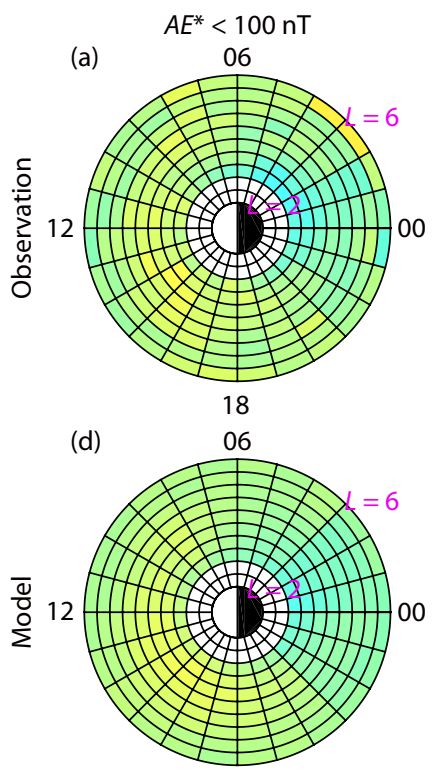

18

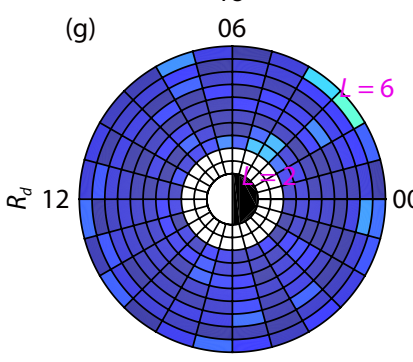

18

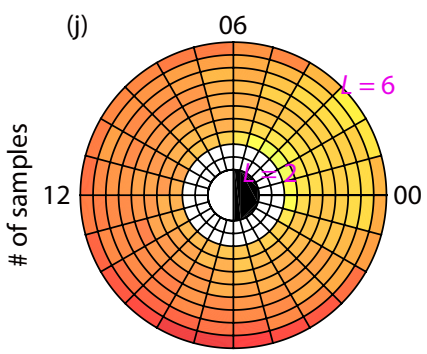

18

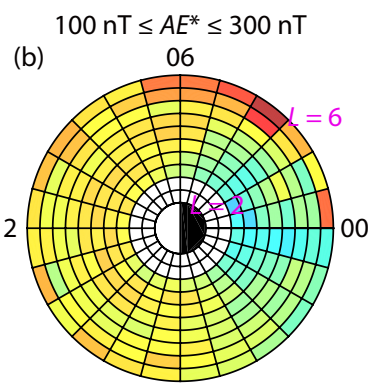

18

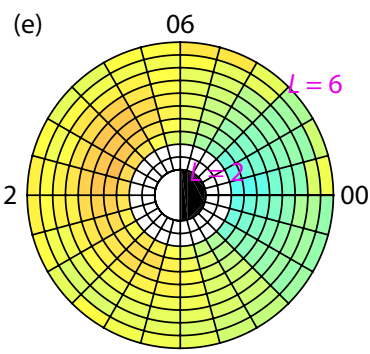

18

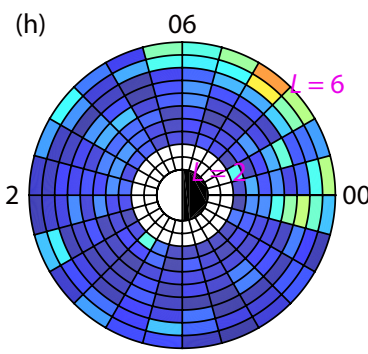

18

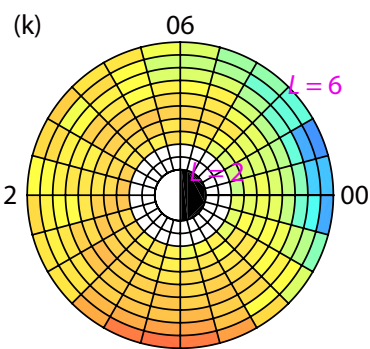

18
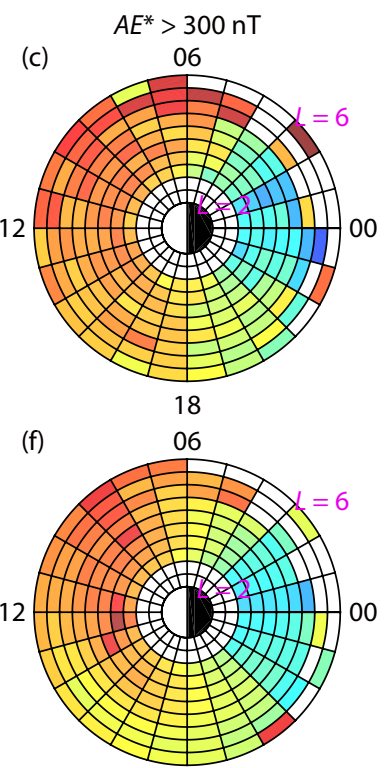

18

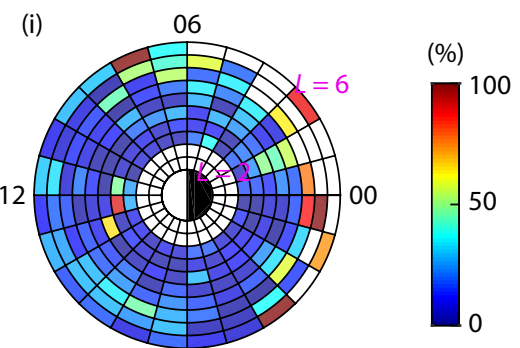

18

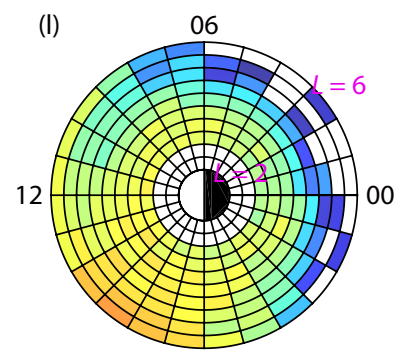

18

Figure 7. (a-c) Global L-MLT distribution of hiss wave amplitude from observations of Database 4; (d-f) model results calculated by Method \#2 of Database $4 ;(\mathrm{g}-\mathrm{i})$ relative differences $\left(R_{d}\right)$ between them; $(\mathrm{j}-\mathrm{l})$ Corresponding numbers of samples during quiet, moderate, and active times (left to right).

\section{Supplementary Materials}

Fitting coefficients of global hiss wave amplitudes described by Equation (1), with $A, B, C$, and $D$ standing for $L / 10, \mathrm{MLT} / 24$, $\mathrm{MLAT} / 20$, and $A E^{*} / 500$ respectively in the tables below.

\section{References}

Albert, J. M. (1994). Quasi-linear pitch angle diffusion coefficients: Retaining high harmonics. Journal of Geophysical Research, 99(A12), 23,741-23,745. https://doi.org/10.1029/94JA02345

Abel, B., and Thorne, R. M. (1998a). Electron scattering loss in Earth's inner magnetosphere: 1. dominant physical processes. J. Geophys. Res. Space Phys., 103(A2), 2385-2396. https://doi.org/10.1029/97JA02919

Abel, B., and Thorne, R. M. (1998b). Electron scattering loss in Earth's inner magnetosphere: 2 . sensitivity to model parameters. J. Geophys. Res. Space Phys., 103(A2), 2397-2407. https://doi.org/10.1029/97JA02920

Agapitov, O., Artemyev, A., Krasnoselskikh, V., Khotyaintsev, Y. V., Mourenas, D., Breuillard, H., Balikhin, M., and Rolland, G. (2013). Statistics of whistler mode waves in the outer radiation belt: Cluster STAFF-SA measurements. J. Geophys. Res. Space Phys., 118(6), 3407-3420.

https://doi.org/10.1002/jgra.50312

Agapitov, O. V., Artemyev, A. V., Mourenas, D., Kasahara, Y., and Krasnoselskikh, V. (2014). Inner belt and slot region electron lifetimes and energization rates based on AKEBONO statistics of whistler waves. J. Geophys. Res. Space Phys., 119(4), 2876-2893. https://doi.org/10.1002/2014JA019886

Bortnik, J., Thorne, R. M., and Meredith, N. P. (2008). The unexpected origin of plasmaspheric hiss from discrete chorus emissions. Nature, 452(7183), 62-66. https://doi.org/10.1038/nature06741

He, F., Zhang, X. X., Chen, B., and Fok, M. C. (2011). Reconstruction of the 


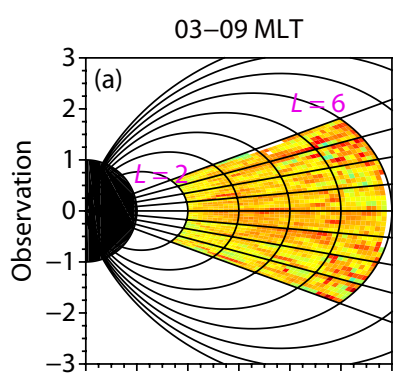

Database 4: 2012/9/8-2017/6/30 (even number of days)
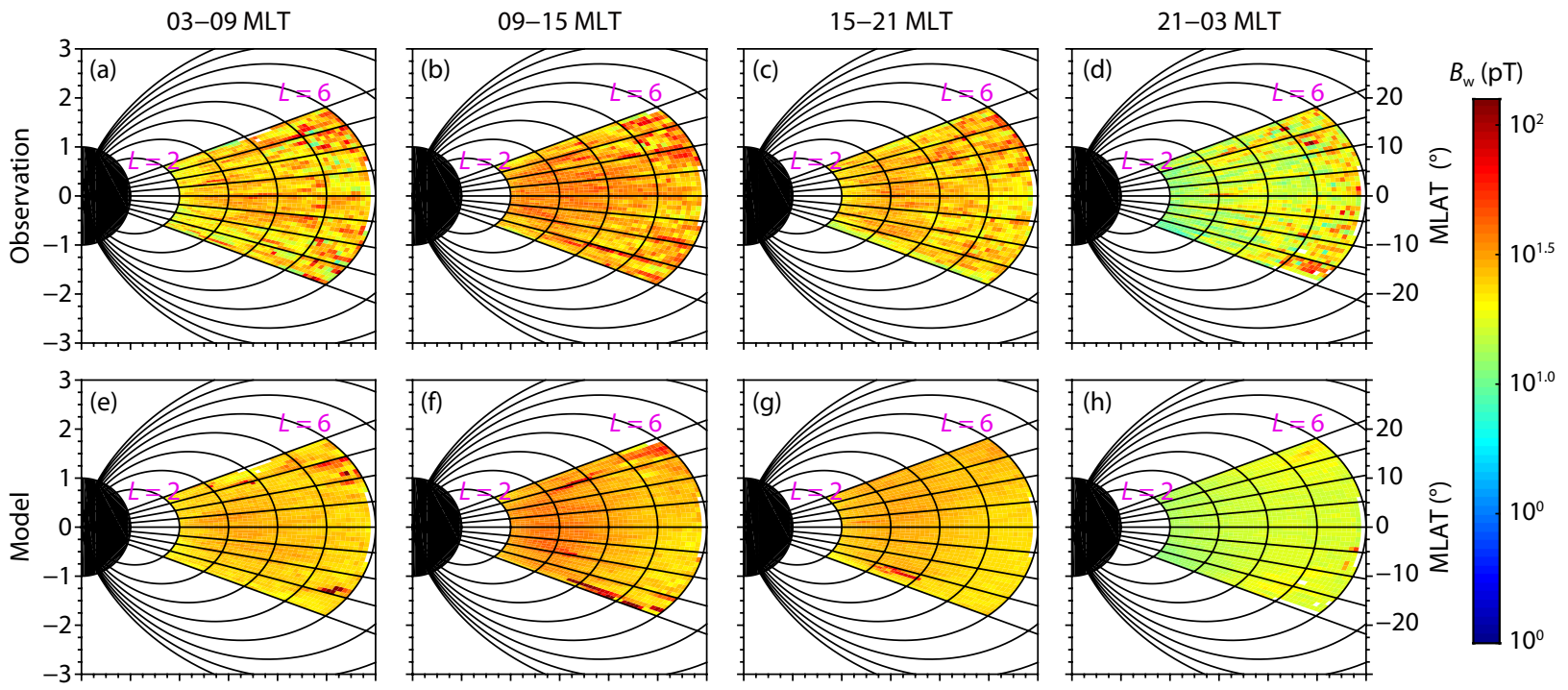

$10^{1.0}$
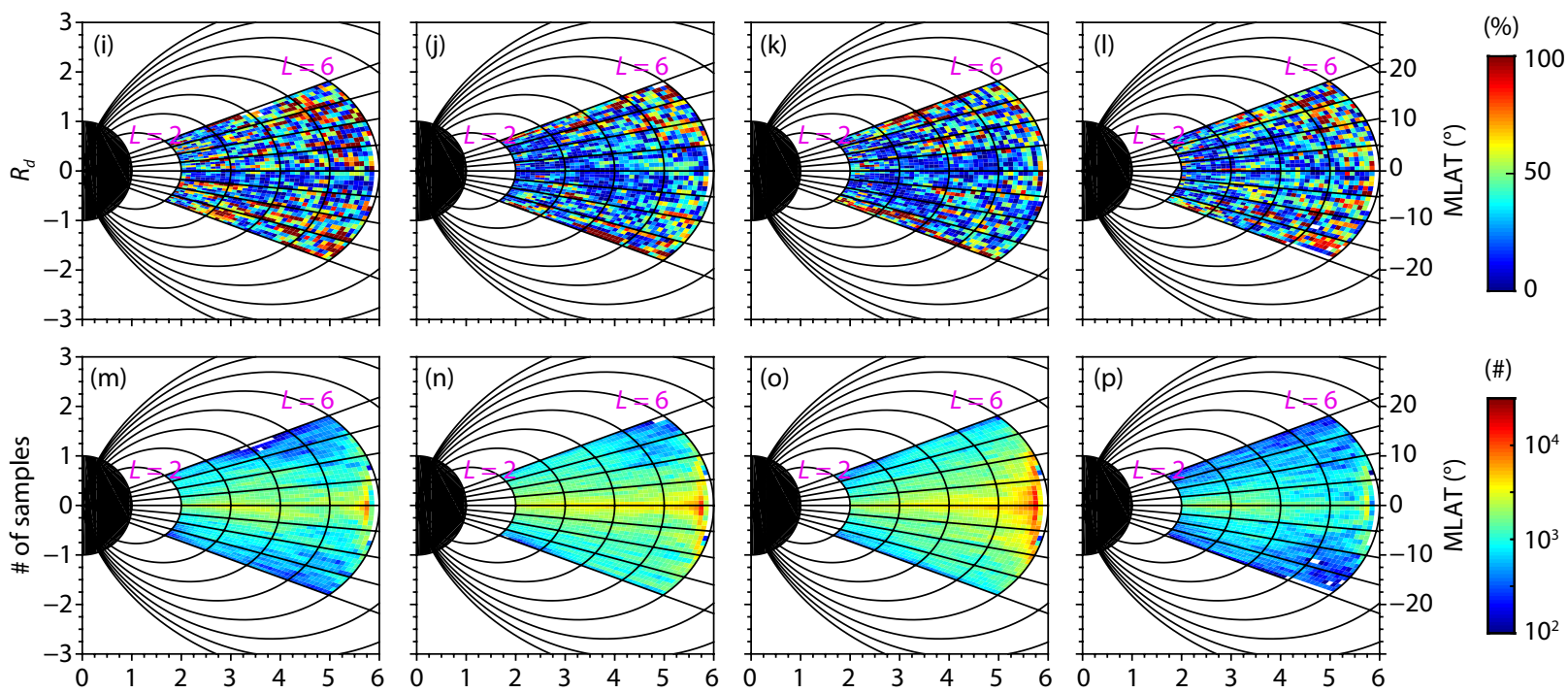

Figure 8. (a-d) Latitudinal L-MLAT distribution of hiss wave amplitude from observations in Database 4; (e-h) model results calculated by Method \#2 of Database 4 ; $(\mathrm{i}-\mathrm{l})$ relative differences $\left(R_{d}\right)$ between them; $(\mathrm{m}-\mathrm{p})$ corresponding numbers of samples during different MLT sectors (left to right).

Table S1. For the case of $3 \leq \mathrm{MLT}<9$ when $A E^{*}<100 \mathrm{nT}$.

\begin{tabular}{ccccc}
\hline & $A^{3}$ & $A^{2}$ & $A$ & 1 \\
\hline$B^{2} C^{2} D^{2}$ & -266514.9971 & 396133.7612 & -184520.1083 & 22417.0169 \\
$B^{2} C^{2} D$ & 54078.0900 & -73746.8387 & 34075.0324 & -4159.9805 \\
$B^{2} C^{2}$ & -5621.4548 & 6523.7330 & -2538.6389 & 289.3744 \\
$B^{2} C D^{2}$ & 711003.3528 & -869701.6097 & 336135.7473 & -36131.3944 \\
$B^{2} C D$ & -162514.4515 & 198471.9394 & -77133.7749 & 8403.3444 \\
$B^{2} C$ & 5460.4956 & -7038.6107 & 2935.1354 & -339.9128 \\
$B^{2} D^{2}$ & 12383.3494 & 36038.8107 & -26108.7679 & 4090.9090 \\
$B^{2} D$ & -5046.8114 & -11409.0749 & 8713.9449 & -1313.8818 \\
$B^{2}$ & 1167.8761 & -144.9262 & -286.1891 & 57.2291 \\
$B C^{2} D^{2}$ & 109462.4900 & -189592.8830 & 95759.0780 & -12030.0396 \\
\hline
\end{tabular}


Continued from Table S1

\begin{tabular}{|c|c|c|c|c|}
\hline & $A^{3}$ & $A^{2}$ & $A$ & 1 \\
\hline$B C^{2} D$ & -22770.5610 & 35900.9413 & -17996.1522 & 2267.7925 \\
\hline$B C^{2}$ & 2614.9908 & -3200.3276 & 1298.0470 & -150.7319 \\
\hline$B C D^{2}$ & -399735.7029 & 486461.0926 & -185965.6797 & 19759.4410 \\
\hline$B C D$ & 89648.4882 & -108883.2325 & 41859.1725 & -4512.8917 \\
\hline$B C$ & -3072.1548 & 3905.0191 & -1596.3226 & 181.6559 \\
\hline$B D^{2}$ & 13119.3847 & -34517.3428 & 17091.5814 & -2350.3062 \\
\hline$B D$ & -3057.1654 & 10660.3297 & -5661.1607 & 768.2433 \\
\hline$B$ & -133.1410 & -385.0074 & 285.6188 & -41.8407 \\
\hline$C^{2} D^{2}$ & -8925.5333 & 19872.9552 & -11002.8916 & 1400.9854 \\
\hline$C^{2} D$ & 1961.5531 & -3790.8888 & 2059.6952 & -260.8511 \\
\hline$c^{2}$ & -260.5572 & 337.9240 & -141.9811 & 16.4166 \\
\hline$C D^{2}$ & 49165.7159 & -59712.5852 & 22671.5047 & -2376.0183 \\
\hline$C D$ & -10827.3537 & 13107.7268 & -4999.7083 & 531.5406 \\
\hline$C$ & 374.1982 & -471.3872 & 189.8032 & -21.1261 \\
\hline$D^{2}$ & -3683.7443 & 5466.8612 & -2190.9823 & 278.3865 \\
\hline$D$ & 1167.1453 & -1918.8436 & 821.3382 & -101.4842 \\
\hline 1 & -47.9098 & 104.0285 & -49.1017 & 7.6187 \\
\hline
\end{tabular}

Table S2. For $3 \leq \mathrm{MLT}<9$ when $100 \mathrm{nT} \leq A E^{*} \leq 300 \mathrm{nT}$.

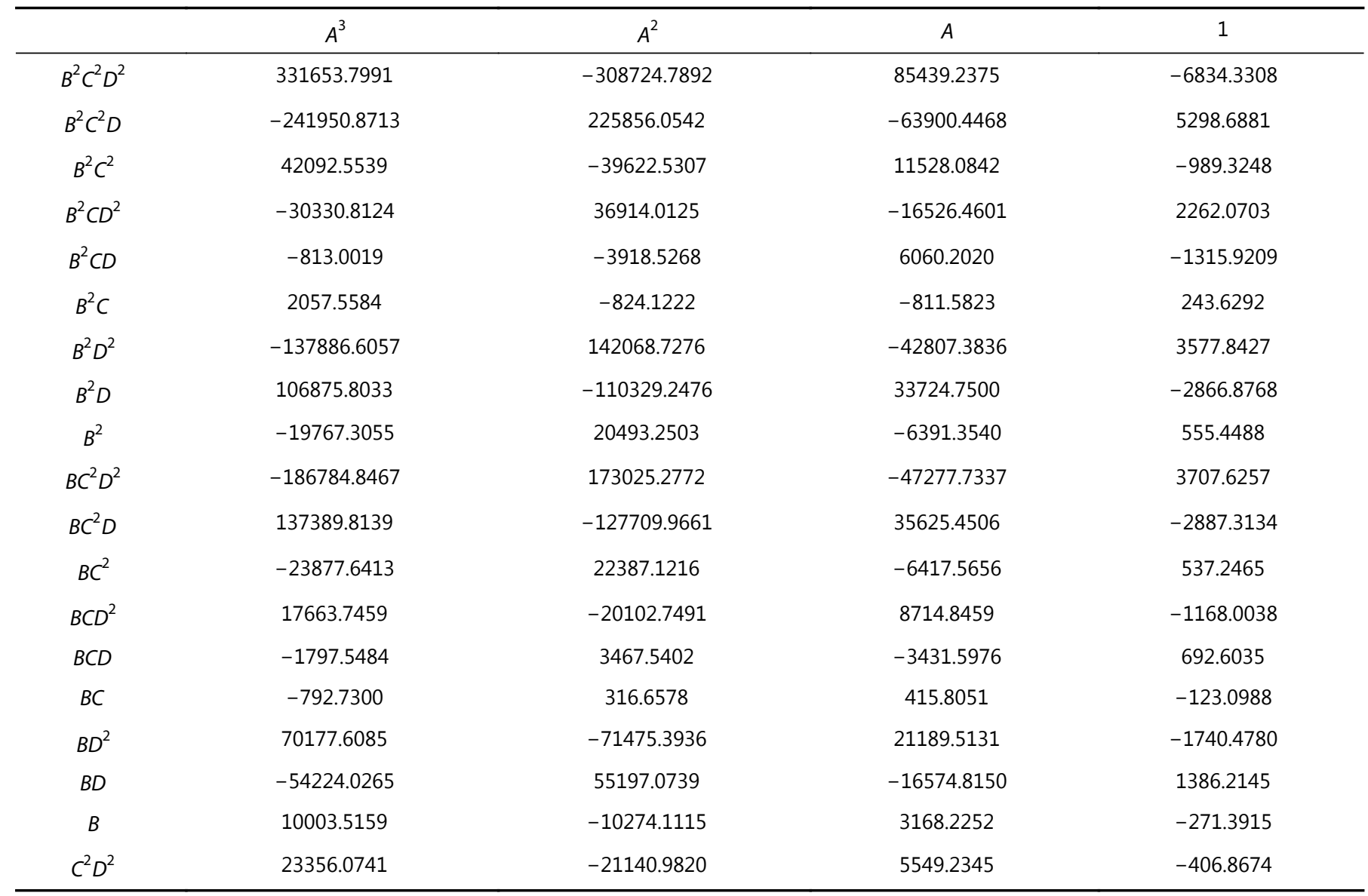


Continued from Table S2

\begin{tabular}{ccccc}
\hline & $A^{3}$ & $A^{2}$ & $A$ & 1 \\
\hline$C^{2} D$ & -17446.3873 & 15895.2195 & -4271.5491 & 324.4526 \\
$C^{2}$ & 3086.8747 & -2847.3167 & 790.1641 & -62.5019 \\
$C D^{2}$ & -1941.2948 & 2015.7225 & -843.7662 & 110.3938 \\
$C D$ & 311.1382 & -340.1256 & 319.5751 & -65.1887 \\
$C$ & 74.4323 & -51.4628 & -31.4615 & 11.3554 \\
$D^{2}$ & -7709.1922 & 7698.6539 & -2219.8704 & 175.7890 \\
$D$ & 6041.5387 & -6004.5814 & 1750.7038 & -141.2786 \\
1 & -1111.2043 & 1114.1224 & -333.5586 & 29.1836 \\
\hline
\end{tabular}

Table S3. For $3 \leq \mathrm{MLT}<9$ when $A E^{*}>300 \mathrm{nT}$.

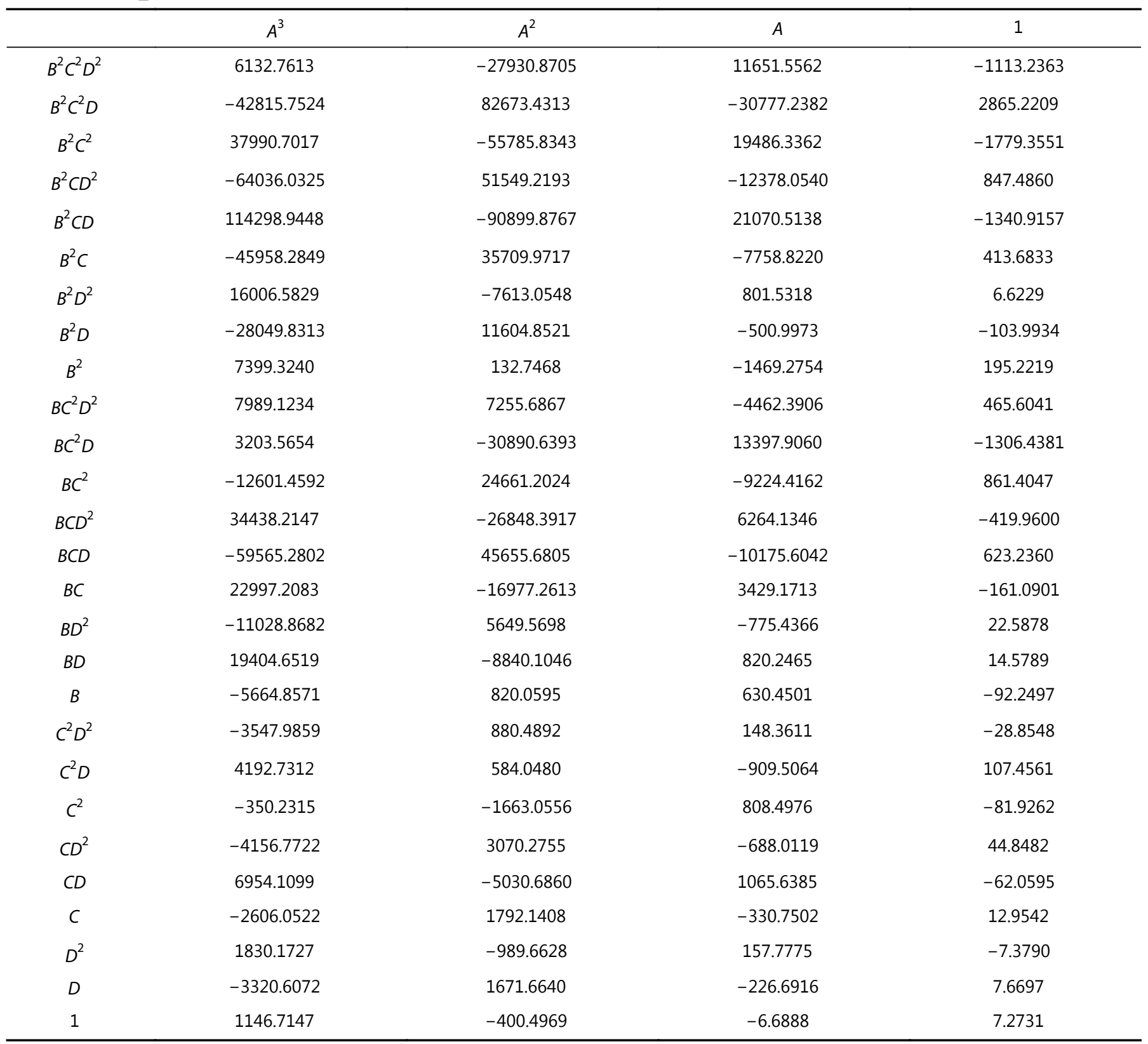


Table S4. For $9 \leq \mathrm{MLT}<15$ when $A E^{*}<100 \mathrm{nT}$.

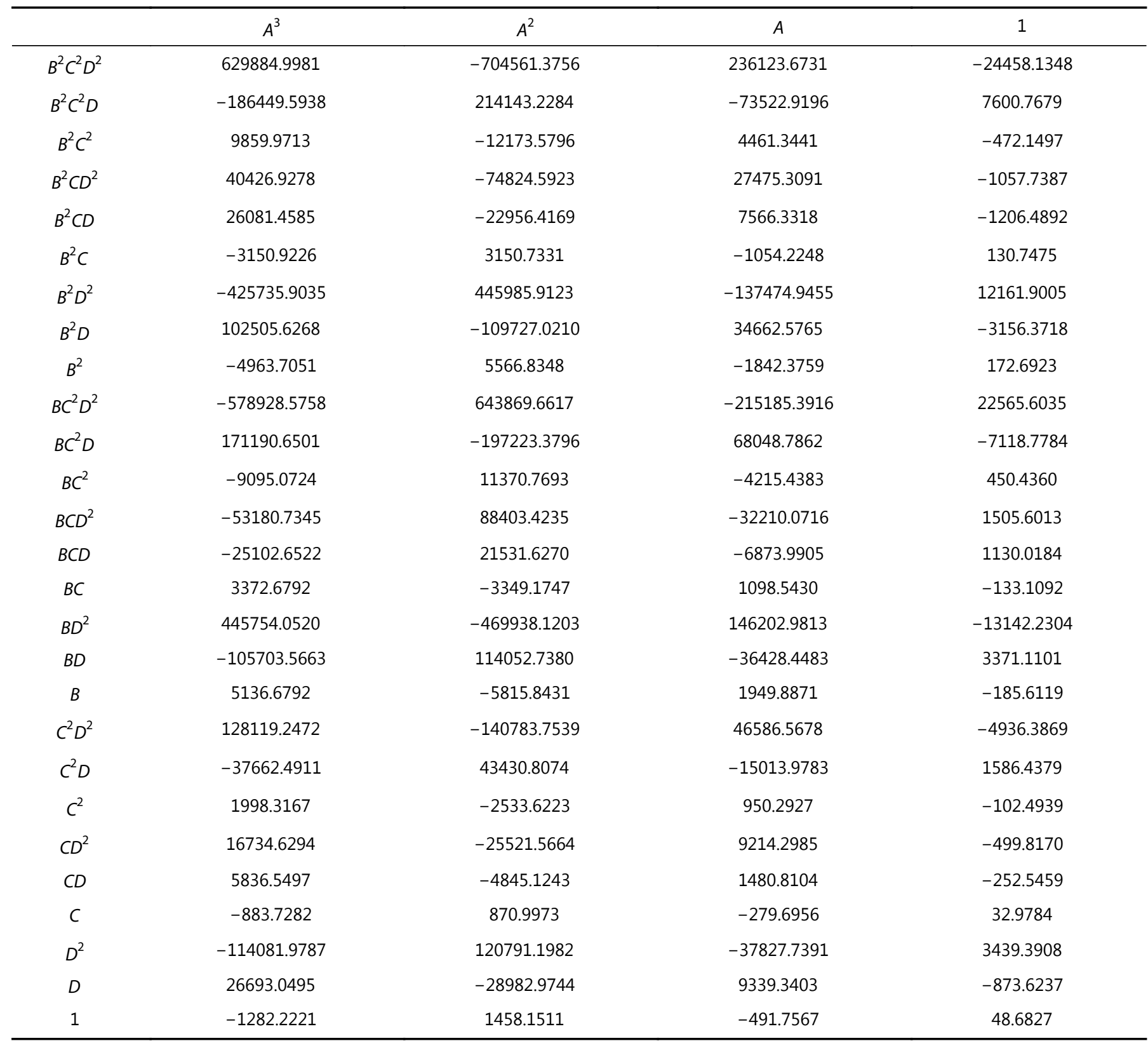

Table S5. For $9 \leq \mathrm{MLT}<15$ when $100 \mathrm{nT} \leq A E^{*} \leq 300 \mathrm{nT}$.

\begin{tabular}{ccccc}
\hline & $A^{3}$ & $A^{2}$ & $A$ & 1 \\
\hline$B^{2} C^{2} D^{2}$ & -169864.1685 & 147455.0384 & -32886.8818 & 1505.1670 \\
$B^{2} C^{2} D$ & 130383.1144 & -112560.8228 & 24526.2227 & -962.8418 \\
$B^{2} C^{2}$ & -22365.0240 & 19117.4984 & -3981.7294 & 114.3034 \\
$B^{2} C D^{2}$ & -139011.8288 & 145722.3046 & -48273.5030 & 4794.3010 \\
$B^{2} C D$ & 115734.3602 & -122340.2537 & 40873.8247 & -4056.8831 \\
$B^{2} C$ & -21231.0770 & 22556.7890 & -7555.3091 & 740.8990 \\
$B^{2} D^{2}$ & -37444.2493 & 48027.6866 & -18563.9424 & 1952.8074 \\
$B^{2} D$ & 26352.4851 & -34065.8492 & 13311.0788 & -1439.8912 \\
$B^{2}$ & -4917.4746 & 6152.4054 & -2368.9573 & 257.3706 \\
\hline
\end{tabular}


Continued from Table S5

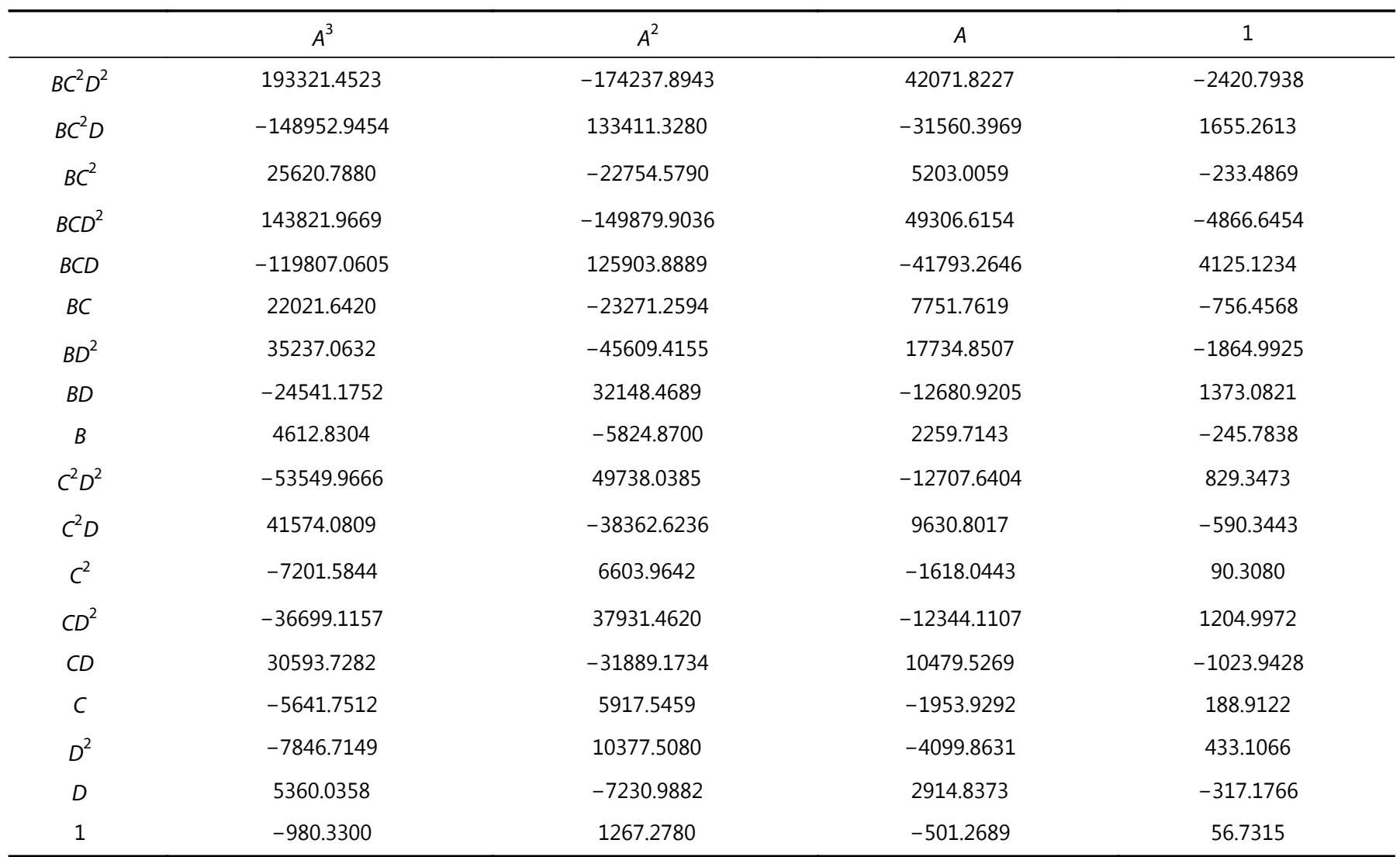

Table S6. For $9 \leq \mathrm{MLT}<15$ when $A E^{*}>300 \mathrm{nT}$.

\begin{tabular}{|c|c|c|c|c|}
\hline & $A^{3}$ & $A^{2}$ & $A$ & 1 \\
\hline$B^{2} C^{2} D$ & 65046.8285 & -76045.8471 & 26740.9094 & -2483.7590 \\
\hline$B^{2} C^{2}$ & -29375.6610 & 35116.2003 & -12477.5683 & 1147.1708 \\
\hline$B^{2} C D^{2}$ & -150.4137 & -931.4761 & 888.6293 & -135.8866 \\
\hline$B^{2} C$ & -15577.0949 & 14709.2991 & -3816.5509 & 263.8378 \\
\hline$B^{2} D^{2}$ & 1501.7008 & -2734.6008 & 1196.6681 & -122.1471 \\
\hline$B^{2} D$ & -5178.1268 & 8533.7132 & -3533.0662 & 353.4630 \\
\hline$B^{2}$ & 3170.0384 & -5116.3017 & 2086.0565 & -209.4092 \\
\hline$B C^{2}$ & 28769.8107 & -34819.4777 & 12474.0677 & -1155.9478 \\
\hline$B C D^{2}$ & -775.3958 & 2061.5781 & -1276.5211 & 171.4736 \\
\hline$B C D$ & -15580.0601 & 13246.3806 & -2720.2183 & 111.5433 \\
\hline$B C$ & 14743.2103 & -13635.5241 & 3438.1251 & -227.0693 \\
\hline$B D^{2}$ & -1534.6187 & 2744.9817 & -1183.0081 & 119.6319 \\
\hline$B D$ & 5378.4352 & -8676.4002 & 3526.4529 & -348.9818 \\
\hline$B$ & -3102.6496 & 5018.5670 & -2032.9596 & 202.7243 \\
\hline$C^{2} D^{2}$ & -8149.1462 & 9285.7846 & -3200.7904 & 293.8390 \\
\hline
\end{tabular}


Continued from Table S6

\begin{tabular}{ccccc}
\hline & $A^{3}$ & $A^{2}$ & $A$ & 1 \\
\hline$C^{2} D$ & 16073.3979 & -18981.3168 & 6694.7619 & -624.9779 \\
$C^{2}$ & -6818.1265 & 8377.6824 & -3026.2587 & 282.4056 \\
$C D^{2}$ & 472.4027 & -830.8122 & 420.3314 & -51.8246 \\
$C D$ & 3289.2412 & -2608.3565 & 448.6455 & -6.4834 \\
$C$ & -3397.5641 & 3064.5458 & -743.7383 & 45.6803 \\
$D^{2}$ & 411.2663 & -703.0306 & 293.8160 & -29.3386 \\
$D$ & -1452.1749 & 2246.5070 & -885.0933 & 86.5118 \\
1 & 830.0756 & -1301.6374 & 516.5562 & -49.1952 \\
\hline
\end{tabular}

Table S7. For $15 \leq \mathrm{MLT}<21$ when $A E^{*}<100 \mathrm{nT}$.

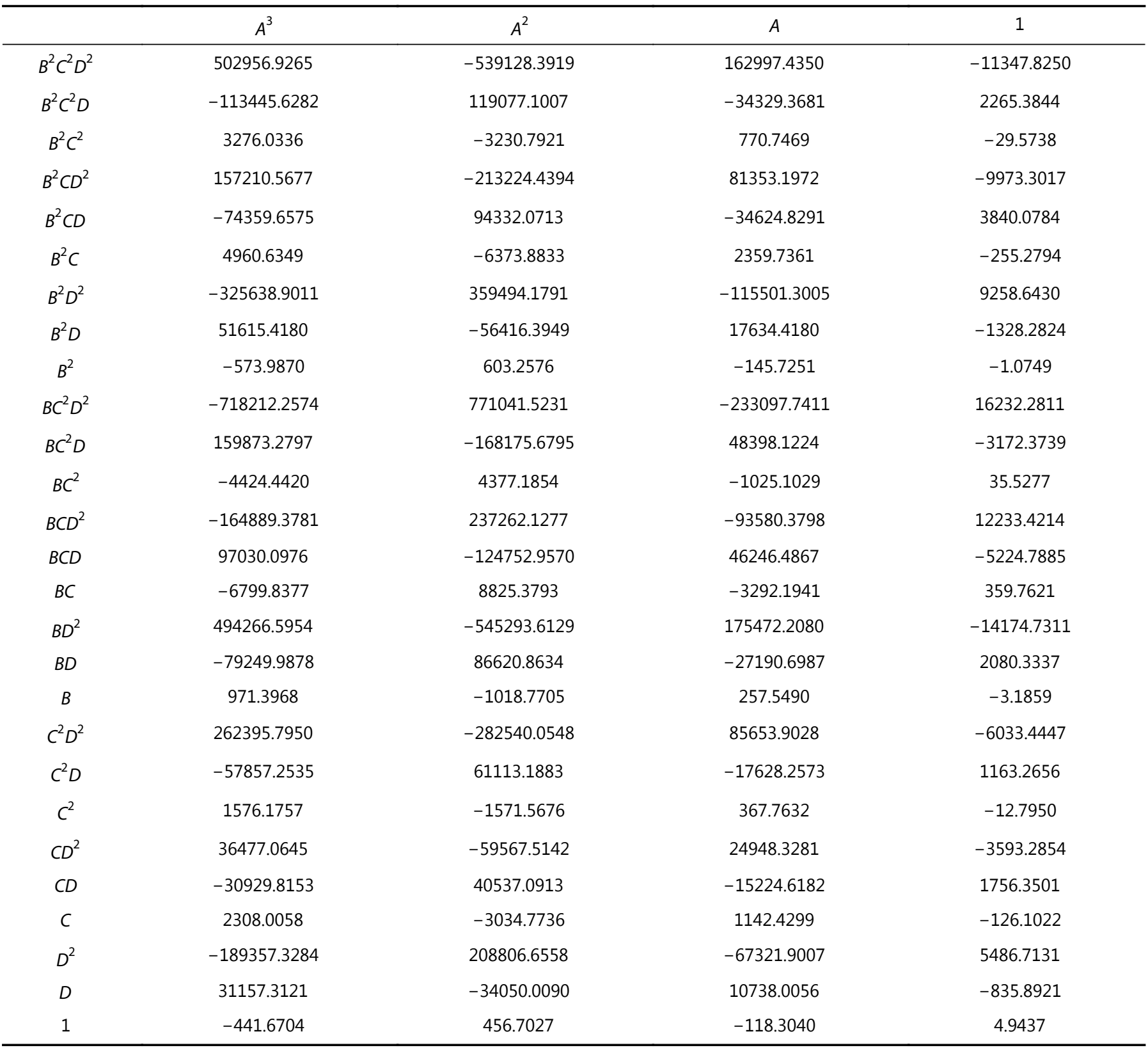


Table S8. For $15 \leq \mathrm{MLT}<21$ when $100 \mathrm{nT} \leq A E^{*} \leq 300 \mathrm{nT}$.

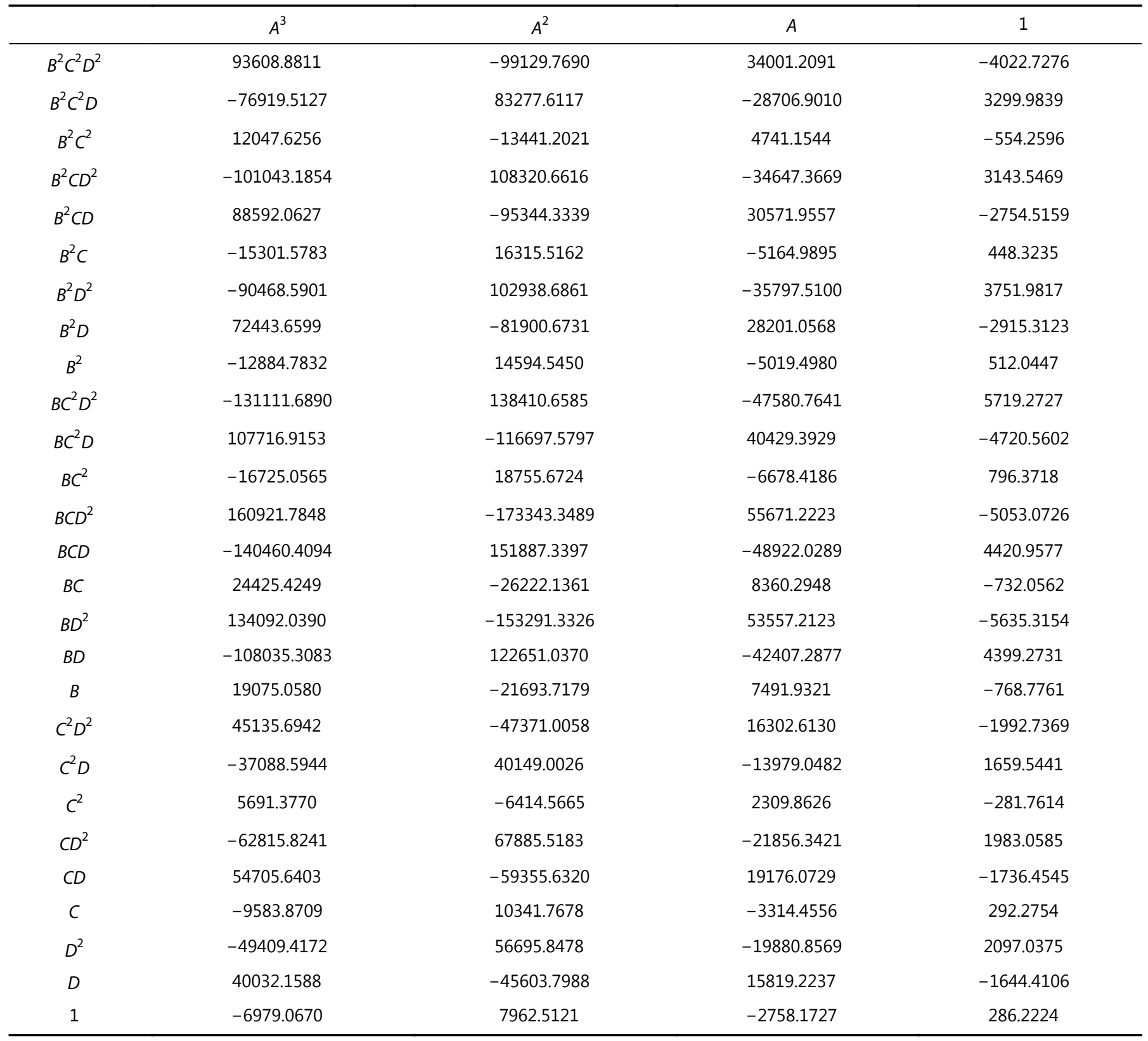

Table S9. For $15 \leq \mathrm{MLT}<21$ when $A E^{*}>300 \mathrm{nT}$.

\begin{tabular}{ccccc}
\hline & $A^{3}$ & $A^{2}$ & $A$ & 1 \\
\hline$B^{2} C^{2} D^{2}$ & -1829.3591 & -587.4156 & 1164.1513 & -259.9248 \\
$B^{2} C^{2} D$ & -1757.2425 & 4277.4628 & -3162.8914 & 686.3738 \\
$B^{2} C^{2}$ & -3003.5657 & 3324.1760 & -371.8121 & -184.5379 \\
$B^{2} C D^{2}$ & -12211.0051 & 10877.9200 & -2708.4724 & 203.9189 \\
$B^{2} C D$ & 34058.6401 & -32021.0297 & 8632.3466 & -686.0533 \\
$B^{2} C$ & -15231.1783 & 14413.9471 & -3857.5838 & 297.3967 \\
$B^{2} D^{2}$ & 4236.7924 & -3758.4586 & 941.0589 & -45.7887 \\
$B^{2} D$ & -6926.4342 & 6521.6285 & -1678.5517 & 61.2233 \\
$B^{2}$ & 2985.1491 & -3203.8975 & 910.2741 & -37.0109 \\
\hline
\end{tabular}


Continued from Table S9

\begin{tabular}{|c|c|c|c|c|}
\hline & $A^{3}$ & $A^{2}$ & $A$ & 1 \\
\hline$B C^{2} D^{2}$ & 700.9561 & 2672.3186 & -2260.9915 & 430.0345 \\
\hline$B C^{2} D$ & 5060.9111 & -8754.0784 & 5522.8809 & -1092.7459 \\
\hline$B C^{2}$ & 3854.3675 & -4227.9542 & 245.1139 & 304.1879 \\
\hline$B C D^{2}$ & 17249.2805 & -15189.3713 & 3705.5840 & -272.1701 \\
\hline$B C D$ & -48633.6842 & 45430.6074 & -12127.1988 & 952.8634 \\
\hline$B C$ & 21845.9720 & -20534.2901 & 5436.1308 & -413.5728 \\
\hline$B D^{2}$ & -5921.7142 & 5368.5874 & -1374.5254 & 69.1577 \\
\hline$B D$ & 10043.8037 & -9731.4713 & 2574.3572 & -104.3332 \\
\hline$B$ & -4786.1755 & 5257.6537 & -1535.5133 & 73.1924 \\
\hline$C^{2} D^{2}$ & 224.8326 & -1408.1570 & 963.8340 & -169.6245 \\
\hline$C^{2} D$ & -2273.1720 & 3649.4858 & -2214.6980 & 420.1827 \\
\hline$c^{2}$ & -1467.1437 & 1555.0067 & -47.5418 & -117.9417 \\
\hline$C D^{2}$ & -6012.4862 & 5221.5247 & -1241.6993 & 88.5275 \\
\hline$C D$ & 17125.1575 & -15871.8048 & 4184.9588 & -324.8277 \\
\hline$C$ & -7718.2414 & 7195.9406 & -1879.5879 & 141.0361 \\
\hline$D^{2}$ & 2083.5260 & -1928.0878 & 503.1514 & -26.1625 \\
\hline$D$ & -3685.4162 & 3651.8279 & -984.5011 & 43.4187 \\
\hline 1 & 1962.9860 & -2184.9943 & 650.9175 & -33.2332 \\
\hline
\end{tabular}

Table S10. For $21 \leq \mathrm{MLT}<3$ when $A E^{*}<100 \mathrm{nT}$.

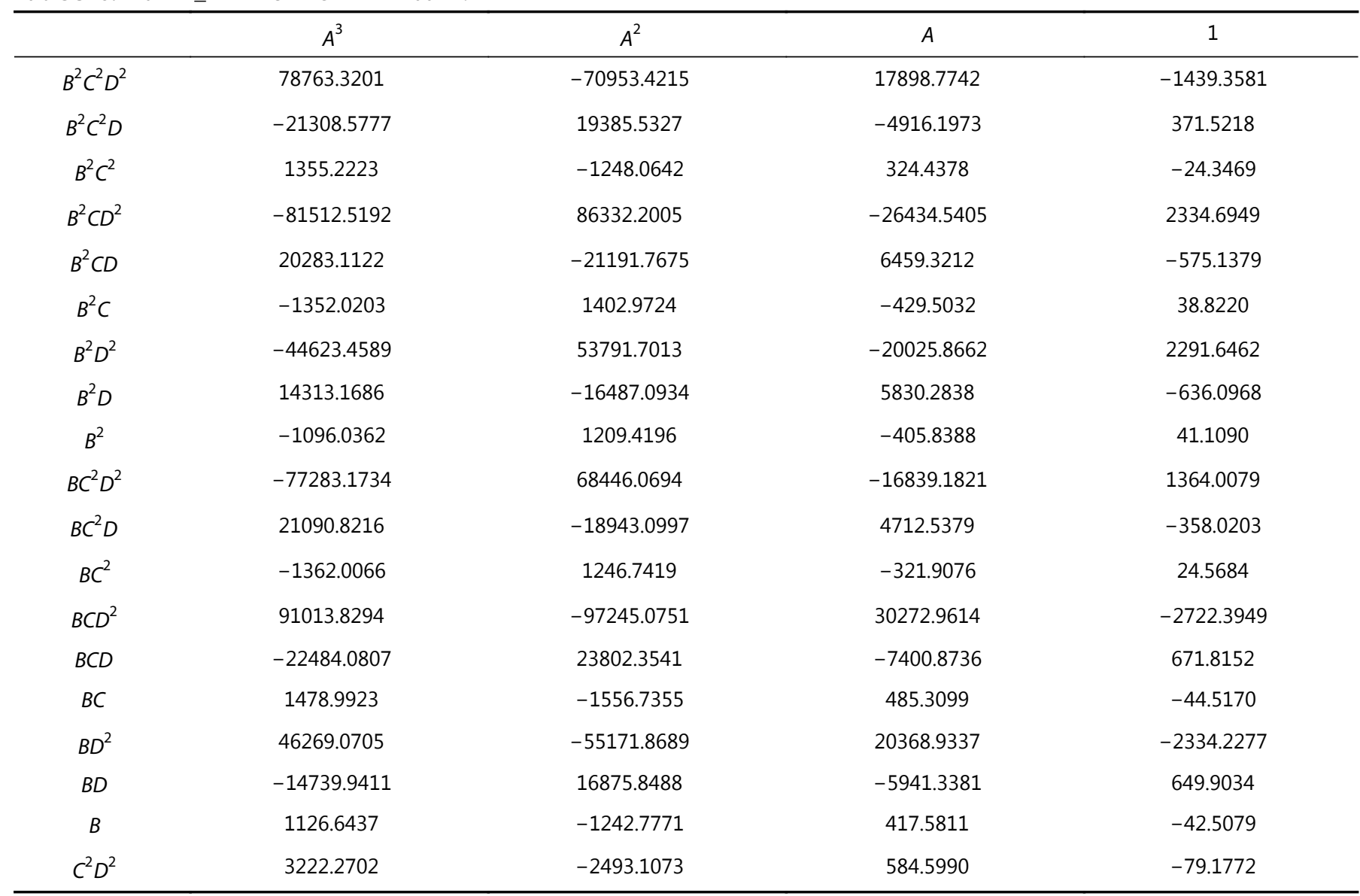


Continued from Table S10

\begin{tabular}{ccccc}
\hline & $A^{3}$ & $A^{2}$ & $A$ & 1 \\
\hline$C^{2} D$ & -1104.8296 & 964.6767 & -251.1849 & 27.5374 \\
$C^{2}$ & 75.8759 & -68.7066 & 18.7087 & -1.9568 \\
$C D^{2}$ & -10859.7647 & 12081.3927 & -4078.3578 & 401.0778 \\
$C D$ & 2518.3306 & -2831.8652 & 970.0610 & -97.1894 \\
$C$ & -147.7759 & 165.3412 & -56.4296 & 5.6820 \\
$D^{2}$ & -1896.6714 & 2283.9892 & -893.5652 & 119.4325 \\
$D$ & 677.7034 & -764.1779 & 272.3790 & -33.1051 \\
1 & -51.6867 & 54.9470 & -17.4602 & 3.2163 \\
\hline
\end{tabular}

Table S11. For $21 \leq \mathrm{MLT}<3$ when $100 \mathrm{nT} \leq A E^{*} \leq 300 \mathrm{nT}$.

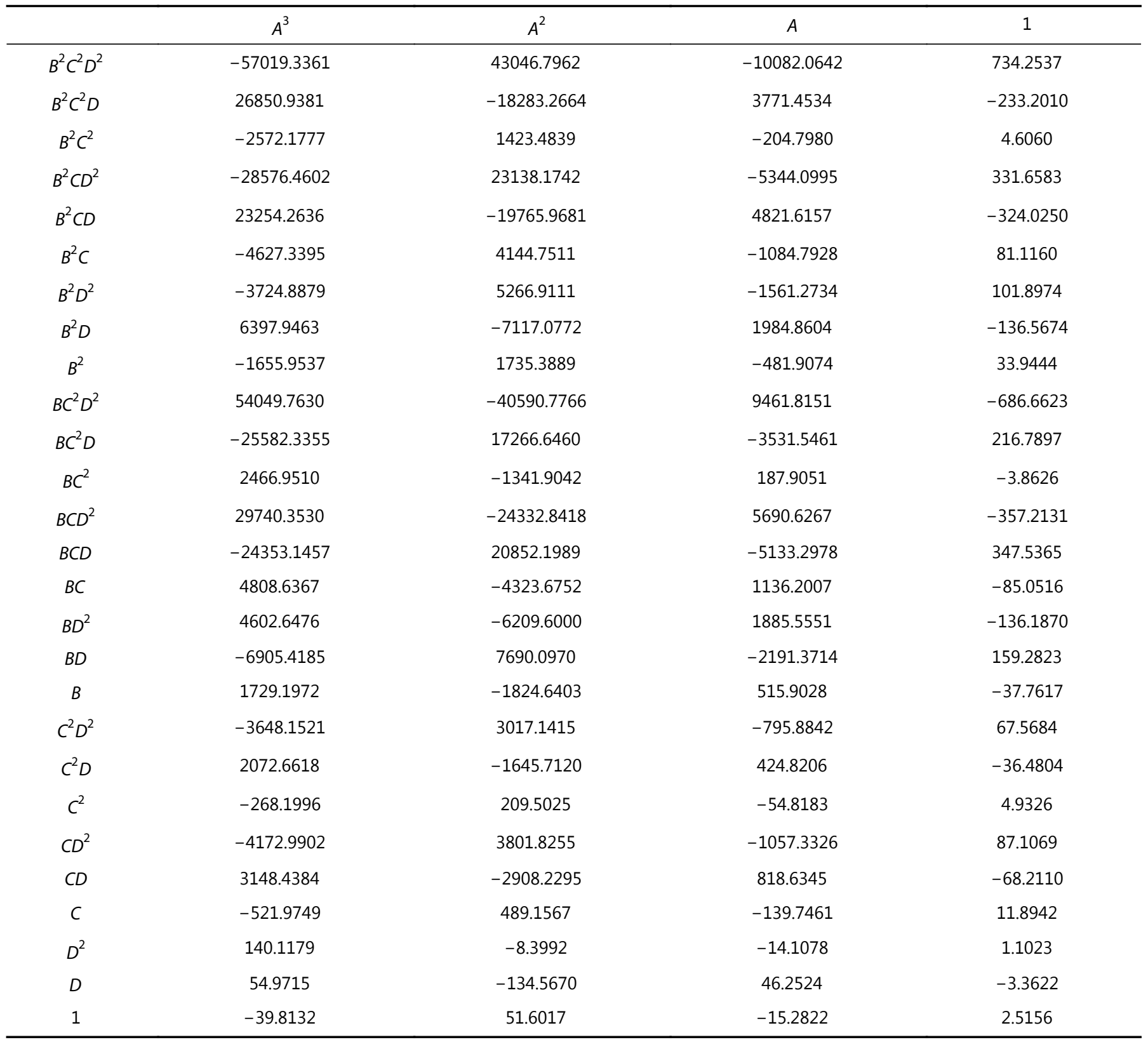


Table S12. For $21 \leq \mathrm{MLT}<3$ when $A E^{*}>300 \mathrm{nT}$.

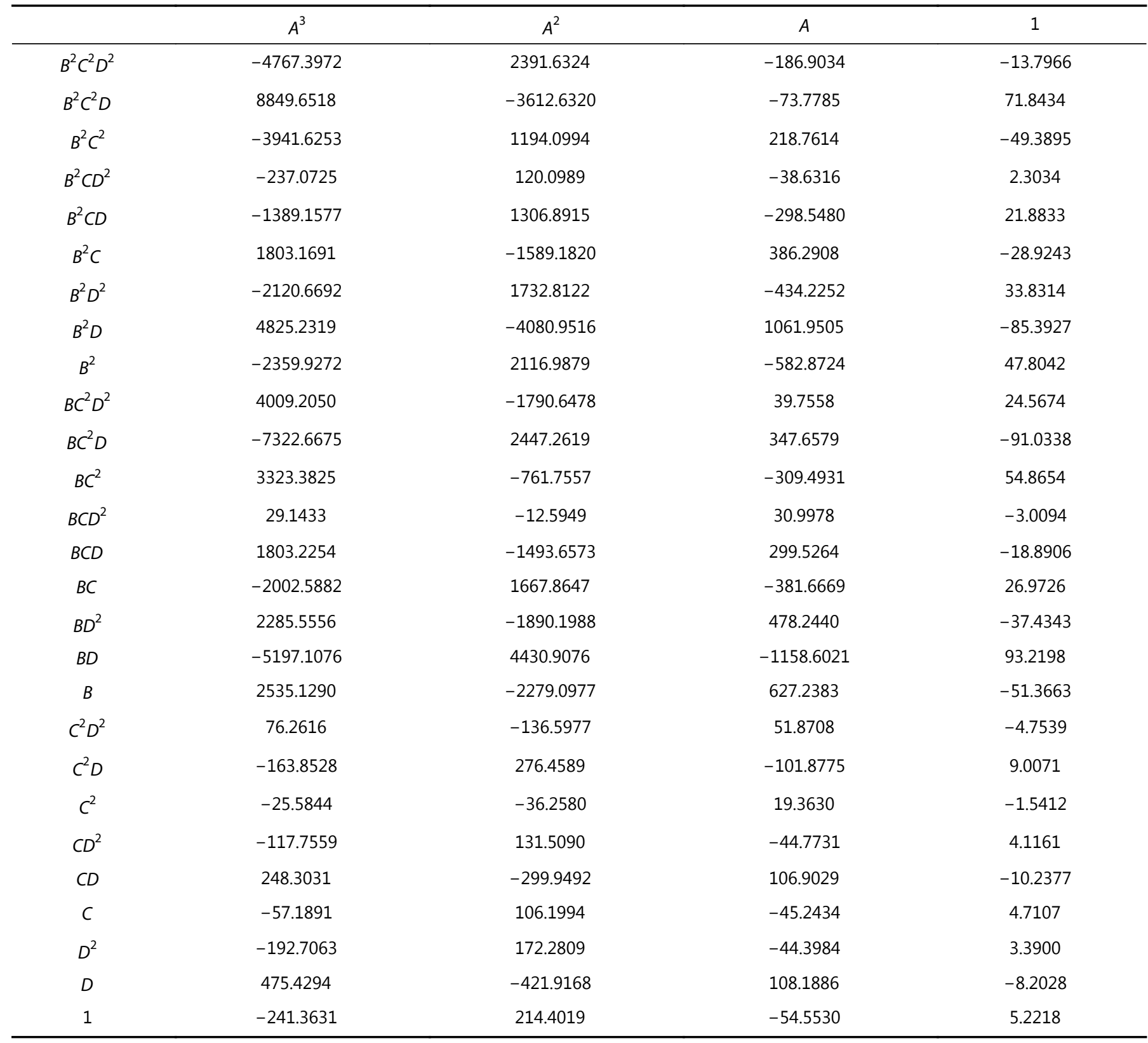

plasmasphere from Moon-based EUV images. J. Geophys. Res. Space Phys., 116(A11), A11203. https://doi.org/10.1029/2010JA016364

He, F., Zhang, X. X., Chen, B., Fok, M. C., and Zou, Y. L. (2013). Moon-based EUV imaging of the Earth's plasmasphere: Model simulations. J. Geophys. Res. Space Phys., 118(11), 7085-7103. https://doi.org/10.1002/2013JA018962

He, F., Zhang, X. X., Chen, B., Fok, M. C., and Nakano, S. (2016). Determination of the Earth's plasmapause location from the CE-3 EUVC images. J. Geophys. Res. Space Phys., 121(1), 296-304. https://doi.org/10.1002/2015JA021863

He, F., Zhang, X. X., Lin, R. L., Fok, M. C., Katus, R. M., Liemohn, M. W., Gallagher, D. L., and Nakano, S. (2017). A new solar wind-driven global dynamic plasmapause model: 2. Model and validation. J. Geophys. Res. Space Phys., 122(7), 7172-7187. https://doi.org/10.1002/2017JA023913

Hua, M., Ni, B. B., Li, W., Gu, X. D., Fu, S., Shi, R., Xiang, Z., Cao, X., Zhang, W. X., and Guo, Y. J. (2019). Evolution of radiation belt electron pitch angle distribution due to combined scattering by plasmaspheric hiss and magnetosonic waves. Geophys. Res. Lett., 46(6), 3033-3042. https://doi.org/10.1029/2018GL081828

Katus, R. M., Gallagher, D. L., Liemohn, M. W., Keesee, A. M., and Sarno-Smith, L.
K. (2015). Statistical storm time examination of MLT-dependent plasmapause location derived from IMAGE EUV. J. Geophys. Res. Space Phys, 120(7), 5545-5559. https://doi.org/10.1002/2015JA021225

Kim, K. C., Lee, D. Y., and Shprits, Y. (2015). Dependence of plasmaspheric hiss on solar wind parameters and geomagnetic activity and modeling of its global distribution. J. Geophys. Res. Space Phys., 120(2), 1153-1167. https://doi.org/10.1002/2014JA020687

Kletzing, C. A., Kurth, W. S., Acuna, M., MacDowall, R. J., Torbert, R. B., Averkamp, T., Bodet, D., Bounds, S. R., Chutter, M., ... Tyler, J. (2013). The electric and magnetic field instrument suite and integrated science (EMFISIS) on RBSP. Space Sci. Rev., 179(1-4), 127-181. https://doi.org/10.1007/s11214-0139993-6

Li, L. Y., Cao, J. B., and Zhou, G. C. (2008). Whistler-mode waves modify the highenergy electron slot region and the outer radiation belt. Chinese J. Geophys. (in Chinese), 51(2), 316-324. https://doi.org/10.3321/j.issn:00015733.2008.02.004

Li, W., Thorne, R. M., Bortnik, J., Reeves, G. D., Kletzing, C. A., Kurth, W. S., Hospodarsky, G. B., Spence, H. E., Blake, J. B., ... Thaller, S. A. (2013). An 
unusual enhancement of low-frequency plasmaspheric hiss in the outer plasmasphere associated with substorm-injected electrons. Geophys. Res. Lett., 40(15), 3798-3803. https://doi.org/10.1002/grl.50787

Li, W., Chen, L., Bortnik, J., Thorne, R. M., Angelopoulos, V., Kletzing, C. A., Kurth, W. S., and Hospodarsky, G. B. (2015a). First evidence for chorus at a large geocentric distance as a source of plasmaspheric hiss: Coordinated THEMIS and Van Allen Probes observation. Geophys. Res. Lett., 42(2), 241-248. https://doi.org/10.1002/2014GL062832

Li, W., Ma, Q., Thorne, R. M., Bortnik, J., Kletzing, C. A., Kurth, W. S., Hospodarsky, G. B., and Nishimura, Y. (2015b). Statistical properties of plasmaspheric hiss derived from Van Allen Probes data and their effects on radiation belt electron dynamics. J. Geophys. Res. Space Phys., 120(5), 3393-3405. https://doi.org/10.1002/2015JA021048

Lyons, L. R., Thorne, R. M., and Kennel, C. F. (1972). Pitch-angle diffusion of radiation belt electrons within the plasmasphere. J. Geophys. Res., 77(19), 3455-3474. https://doi.org/10.1029/JA077i019p03455

Lyons, L. R., and Thorne, R. M. (1973). Equilibrium structure of radiation belt electrons. J. Geophys. Res., 78(13), 2142-2149.

https://doi.org/10.1029/JA078i013p02142

Meredith, N. P., Horne, R. B., Thorne, R. M., Summers, D., and Anderson, R. R. (2004). Substorm dependence of plasmaspheric hiss. J. Geophys. Res. Space Phys., 109(A6), A06209. https://doi.org/10.1029/2004JA010387

Meredith, N. P., Horne, R. B., Clilverd, M. A., Horsfall, D., Thorne, R. M., and Anderson, R. R. (2006a). Origins of plasmaspheric hiss. J. Geophys. Res. Space Phys., 111(A9), A09217. https://doi.org/10.1029/2006JA011707

Meredith, N. P., Horne, R. B., Glauert, S. A., Thorne, R. M., Summers, D., Albert, J. M., and Anderson, R. R. (2006b). Energetic outer zone electron loss timescales during low geomagnetic activity. J. Geophys. Res. Space Phys., 111(A5), A05212. https://doi.org/10.1029/2005JA011516

Meredith, N. P., Horne, R. B., Glauert, S. A., and Anderson, R. R. (2007). Slot region electron loss timescales due to plasmaspheric hiss and lightning-generated whistlers. J. Geophys. Res. Space Phys., 112(A8), A08214. https://doi.org/10.1029/2007JA012413

Mosier, S. R., Kaiser, M. L., and Brown, L. W. (1973). Observations of noise bands associated with the upper hybrid resonance by the IMP 6 radio astronomy experiment. J. Geophys. Res., 78(10), 1673-1679. https://doi.org/10.1029/JA078i010p01673

Ni, B. B., Bortnik, J., Thorne, R. M., Ma, Q. L., and Chen, L. J. (2013). Resonant scattering and resultant pitch angle evolution of relativistic electrons by plasmaspheric hiss. J. Geophys. Res. Space Phys., 118(12), 7740-7751. https://doi.org/10.1002/2013JA019260

Ni, B. B., Li, W., Thorne, R. M., Bortnik, J., Ma, Q. L., Chen, L. J., Kletzing, C. A., Kurth, W. S., Hospodarsky, G. B., ... Claudepierre, S. G. (2014). Resonant scattering of energetic electrons by unusual low-frequency hiss. Geophys. Res. Lett., 41(6), 1854-1861. https://doi.org/10.1002/2014GL059389

Ni, B. B., Hua, M., Zhou, R. X., Yi, J., and Fu, S. (2017). Competition between outer zone electron scattering by plasmaspheric hiss and magnetosonic waves. Geophys. Res. Lett., 44(8), 3465-3474. https://doi.org/10.1002/2017GL072989

Ni, B. B., Huang, H., Zhang, W. X., Gu, X. D., Zhao, H., Li, X. L., Baker, D., Fu, S., Xiang, Z., and Cao, X. (2019). Parametric sensitivity of the formation of reversed electron energy spectrum caused by plasmaspheric hiss. Geophys. Res. Lett., 46(8), 4134-4143. https://doi.org/10.1029/2019gl082032

Orlova, K., Spasojevic, M., and Shprits, Y. (2014). Activity-dependent global model of electron loss inside the plasmasphere. Geophys. Res. Lett., 41(11), 3744-3751. https://doi.org/10.1002/2014GL060100

Santolík, O., Parrot, M., Storey, L. R. O., Pickett, J. S., and Gurnett, D. A. (2001). Propagation analysis of plasmaspheric hiss using Polar PWI measurements. Geophys. Res. Lett., 28(6), 1127-1130. https://doi.org/10.1029/2000GL012239
Shi, R., Li, W., Ma, Q. L., Reeves, G. D., Kletzing, C. A., Kurth, W. S., Hospodarsky, G. B., Spence, H. E., Blake, J. B., ... Claudepierre, S. G. (2017). Systematic evaluation of low-frequency hiss and energetic electron injections. J. Geophys. Res. Space Phys., 122(10), 10263-10274. https://doi.org/10.1002/2017JA024571

Smith, E. J., Frandsen, A. M. A., Tsurutani, B. T., Thorne, R. M., and Chan, K. W. (1974). Plasmaspheric hiss intensity variations during magnetic storms. J. Geophys. Res., 79(16), 2507-2510. https://doi.org/10.1029/JA079i016p02507

Spasojevic, M., Shprits, Y. Y., and Orlova, K. (2015). Global empirical models of plasmaspheric hiss using Van Allen Probes. J. Geophys. Res. Space Phys., 120(12), 10370-10383. https://doi.org/10.1002/2015JA021803

Summers, D., Ni, B. B., and Meredith, N. P. (2007a). Timescales for radiation belt electron acceleration and loss due to resonant wave-particle interactions: 1. Theory. J. Geophys. Res. Space Phys., 112(A4), A04206. https://doi.org/10.1029/2006JA011801

Summers, D., Ni, B. B., and Meredith, N. P. (2007b). Timescales for radiation belt electron acceleration and loss due to resonant wave-particle interactions: 2 . Evaluation for VLF chorus, ELF hiss, and electromagnetic ion cyclotron waves. J. Geophys. Res. Space Phys., 112(A4), A04207. https://doi.org/10.1029/2006JA011993

Thorne, R. M., Smith, E. J., Burton, R. K., and Holzer, R. E. (1973). Plasmaspheric hiss. J. Geophys. Res. Space Phys., 78(10), 1581-1596. https://doi.org/10.1029/JA078i010p01581

Thorne, R. M., Smith, E. J., Fiske, K. J., and Church, S. R. (1974). Intensity variation of ELF hiss and chorus during isolated substorms. Geophys. Res. Lett., 1(5), 193-196. https://doi.org/10.1029/GL001i005p00193

Thorne, R. M., Church, S. R., and Gorney, D. J. (1979). On the origin of plasmaspheric hiss: The importance of wave propagation and the plasmapause. J. Geophys. Res. Space Phys., 84(A9), 5241-5247. https://doi.org/10.1029/JA084iA09p05241

Tsurutani, B. T., Falkowski, B. J., Pickett, J. S., Santolik, O., and Lakhina, G. S. (2015). Plasmaspheric hiss properties: Observations from Polar. J. Geophys. Res. Space Phys., 120(1), 414-431. https://doi.org/10.1002/2014JA020518

Verbanac, G., Pierrard, V., Bandić, M., Darrouzet, F., Rauch, J. L., and Décréau, P. (2015). The relationship between plasmapause, solar wind and geomagnetic activity between 2007 and 2011. Ann. Geophys., 33(10), 1271-1283. https://doi.org/10.5194/angeo-33-1271-2015

Xiang, Z., Tan, J. Q., Ni, B. B., Gu, X. D., Cao, X., Zou, Z. Y., Zhou, C., Fu, S., Shi, R., ... Wang, H. (2017). A statistical analysis of the global distribution of plasmaspheric hiss based on Van Allen Probes wave observations. Acta Phys. Sin. (in Chinese), 66(3), 039401. https://doi.org/10.7498/aps.66.039401

Yu, J., Li, L. Y., Cao, J. B., Chen, L., Wang, J., and Yang, J. (2017). Propagation characteristics of plasmaspheric hiss: Van Allen Probe observations and global empirical models. J. Geophys. Res. Space Phys., 122(4), 4156-4167. https://doi.org/10.1002/2016JA023372

Zhang, X. X., He, F., Lin, R. L., Fok, M. C., Katus, R. M., Liemohn, M. W., Gallagher, D. L., and Nakano, S. (2017a). A new solar wind-driven global dynamic plasmapause model: 1. Database and statistics. J. Geophys. Res. Space Phys., 122(7), 7153-7171. https://doi.org/10.1002/2017JA023912

Zhang, X. X., He, F., Chen, B., Shen, C., and Wang, H. N. (2017b). Correlations between plasmapause evolutions and auroral signatures during substorms observed by Chang'e-3 EUV Camera. Earth Planet. Phys., 1(1), 35-43. https://doi.org/10.26464/epp2017005

Zhao, H., Ni, B., Li, X., Baker, D. N., Johnston, W. R., Zhang, W., Xiang, Z., Gu, X., Jaynes, A. N., ... Boyd, A. J. (2019). Plasmaspheric hiss waves generate a reversed energy spectrum of radiation belt electrons. Nat. Phys., 15(4), 367-372. https://doi.org/10.1038/s41567-018-0391-6 